Proceedings of the Edinburgh Mathematical Society (2004) 47, 205-230 (C)

DOI:10.1017/S0013091503000269 Printed in the United Kingdom

\title{
SYSTEMS OF INTEGRAL EQUATIONS WITH WEIGHTED DIFFERENCE KERNELS
}

\author{
D. PORTER ${ }^{1}$ AND N. R. T. BIGGS ${ }^{2}$ \\ ${ }^{1}$ Department of Mathematics, University of Reading, PO Box 220, \\ Whiteknights, Reading RG6 7AX, UK (d.porter@reading.ac.uk) \\ ${ }^{2}$ Department of Mathematics, Keele University, Keele ST5 5BG, UK \\ (nick.biggs@maths.nottingham.ac.uk)
}

(Received 4 April 2003)

\begin{abstract}
Explicit expressions are derived for the inverses of operators of a particular class that includes the operator corresponding to a system of coupled integral equations having weighted difference kernels. The inverses are expressed in terms of a finite number of functions and a systematic way of generating different sets of these functions is devised. The theory generalizes those previously derived for a single integral equation and an integral-equation system with pure difference kernels. The connection is made between the finite generation of inverses and embedding.
\end{abstract}

Keywords: integral equations; finite generation; embedding

2000 Mathematics subject classification: Primary 45A05

\section{Introduction}

Let $\mathcal{A}$ be a given invertible linear operator on a Hilbert space $\mathcal{H}$ equipped with the inner product $(\phi, \psi)$. Suppose that an operator $\mathcal{V}$ can be found with the property

$$
\mathcal{V} \mathcal{A} \phi+\mathcal{A V}^{*} \phi=\sum_{j=1}^{M}\left(\phi, b^{(j)}\right) a^{(j)} \quad(\phi \in \mathcal{H}),
$$

where $M$ is finite, $a^{(j)}$ and $b^{(j)}$ are given elements of $\mathcal{H}$ and the asterisk denotes the adjoint. It follows that

$$
\mathcal{V}^{*} \mathcal{A}^{-1} \phi+\mathcal{A}^{-1} \mathcal{V} \phi=\sum_{j=1}^{M}\left(\phi, d^{(j)}\right) c^{(j)} \quad(\phi \in \mathcal{H}),
$$

where

$$
\mathcal{A} c^{(j)}=a^{(j)}, \quad \mathcal{A}^{*} d^{(j)}=b^{(j)} .
$$

Therefore, provided that (1.2) can be solved for $\mathcal{A}^{-1}$, the inverse operator is expressible in terms of just the elements $c^{(j)}$ and $d^{(j)}$ obtained by solving the $2 M$ equations (1.3). 
This 'finite generation' of inverses was first identified in the context of integral equations by Gohberg and Feldman [6], who investigated the equation

$$
(\mathcal{I}-\mathcal{K}) \phi \equiv \mathcal{A} \phi=f
$$

where $\mathcal{K}$ is a compact operator with a difference kernel $k(x-t)$, by mimicking a parallel structure in the theory of Toeplitz matrices. They showed that $\mathcal{A}^{-1}$ is generated by the solutions of $\mathcal{A} \psi=k$ and $\mathcal{A}^{*} \chi=l$, where $l(x)=\overline{k(-x)}$.

For the same problem, Sakhnovich [13] identified a structure of the form (1.1) by recognizing the connection between the operator $\mathcal{I}-\mathcal{K}$ and the particular Volterra operator $\mathcal{V}$ representing indefinite integration, and he derived an alternative pair of solutions that generate the inverse operator. A generalization of Sakhnovich's work by Porter [11] included the previous generating solutions and derived connections between them and others, dispelling the impression suggested by (1.2) and (1.3) that the functions which determine $\mathcal{A}^{-1}$ are fixed by the representation (1.1). The fact that many pairs of generating solutions may be constructed was established through the development of a structured framework by Porter and Stirling [12], who applied their results to the case in which $\mathcal{K}$ is compact and to the non-compact operator $\mathcal{K}$ generated by the Cauchy singular difference kernel.

Another generalization, by Kon [9], extended the theory of Sakhnovich [13] to include kernels $k(x, t)$ satisfying

$$
\frac{\partial k}{\partial x}+\frac{\partial k}{\partial t}=\sum_{j=1}^{n} M_{j}(x) N_{j}(t),
$$

which are finite-rank departures from difference kernels. Such kernels had previously been investigated by Kailath et al. [7]. Koltracht et al. [8] considered a similar generalization in which the kernel is derived from an integral of a particular form also involving $2 n$ given functions.

Mullikin and Victory [10] extended the original work of Gohberg and Feldman [6] to the case of matrix-valued difference kernels. More recently, Feldman et al. [5] have also considered a system of integral equations with difference kernels and related the generation of the inverse operator to the factorization of a $2 \times 2$ matrix containing the Fourier transform of the kernel.

The present work is concerned with systems of integral equations generated by weighted difference kernels. That is, the kernel of the general operator $\mathcal{K}_{m n}$ in the system is $\kappa_{m n}\left(w_{m} x-w_{n} t\right)$ (with $0 \leqslant x, t \leqslant 1$ ), where $w_{m}$ and $w_{n}$ are real positive constants. It therefore includes matrix-valued operators with pure difference kernels as a special case. The investigation also generalizes that of Porter and Stirling [12] for a single integral equation with a difference kernel by exploring how different forms of the inverse operator may be constructed. This aspect, which has evidently not been examined for a system, even in the pure difference kernel case, is achieved by devising a general transformation between sets of functions that generate the inverse. One particular transformation that is developed to illustrate the theory shows that the inverse operator corresponding to the system of integral equations under consideration is generated by solutions in which 
the forcing terms are the 'boundary values' of the matrix kernel, that is, the values of the kernel at $x=0,1$ and at $t=0,1$. This extends to the weighted difference case corresponding results for pure difference kernels given by Gohberg and Feldman [6] for a single equation and by Mullikin and Victory [10] for a system.

The finite generation of the inverses of integral operators described above is related to so-called embedding properties of the operators. Embedding is a remarkable feature possessed by certain problems containing a parameter, in which the solution for an arbitrary value of that parameter can be expressed in terms of the solutions for finitely many parameter values. This property leads to considerable computational economy when the solution is required across an extended parameter range. Embedding is a particular application of the finite generation of solutions of an integral equation or integralequation system, but it may be exploited without investigating the formation of the inverse operator. Indeed, embedding formulae can exist for operators that are not invertible. Sakhnovich [13] refers briefly to embedding for systems of equations with weighted difference kernels in his extended account of integral equations with difference kernels, using a different approach to that presented here.

Weighted difference kernels may appear to be rather artificial. However, they can arise in the context of certain linear boundary-value problems which, following an application of Green's function theory, lead to sets of coupled integral equations holding on the union of finitely many parallel line segments in the plane, each of finite length. A structured system of equations with weighted difference kernels follows by transforming each of these line segments onto the same (unit) interval. Biggs and Porter [2] encountered such a system in the problem of wave diffraction by a duct, developing and using embedding formulae for this example. It is shown later that a particular choice of the generating functions in the present investigation recovers the formulae derived in the earlier work.

The plan of the paper is to derive the counterparts of (1.1)-(1.3) in the present context and establish various relationships that play a part in the ensuing development of $\S 2$. For example, it is clear from (1.3) that the adjoint of $\mathcal{A}$ is significant and it will satisfy an identity like (1.1). In $\S 3$, the construction of $\mathcal{A}$ from the appropriate formulae of the type (1.1) is developed - a step that explicitly identifies the class of operators under consideration. The construction method is applied to $\mathcal{A}^{-1}$ in $\S 4$, where the general transformation giving different versions of the inverse is devised. The integral-equation system is used to provide concrete examples of the theory in $\S 5$, including a comparison with existing results in the case of pure difference kernels.

\section{Formulation}

Our starting point is the integral-equation system

$$
\lambda_{m} \phi_{m}(x)=f_{m}(x)+\sum_{n=1}^{N} \int_{0}^{1} k_{m n}(x, t) \phi_{n}(t) \mathrm{d} t \quad(0 \leqslant x \leqslant 1, m=1,2, \ldots, N),
$$


which is to be solved for the functions $\phi_{1}(x), \phi_{2}(x), \ldots, \phi_{N}(x)$. It is assumed that the kernels have the forms

$$
k_{m n}(x, t)=\kappa_{m n}\left(w_{m} x-w_{n} t\right),
$$

for some real constants $w_{m}>0$ and some functions $\kappa_{m n}(x)$.

We may write (2.1) in the matrix form:

$$
\Lambda \phi(x)=\boldsymbol{f}(x)+(\mathcal{K} \phi)(x) \quad(0 \leqslant x \leqslant 1),
$$

where $\boldsymbol{\phi}=\left(\phi_{1}, \phi_{2}, \ldots, \phi_{n}\right)^{\mathrm{T}}, \boldsymbol{f}=\left(f_{1}, f_{2}, \ldots, f_{n}\right)^{\mathrm{T}}$ and $\Lambda=\operatorname{diag}\left(\lambda_{1}, \lambda_{2}, \ldots, \lambda_{n}\right)$. Here $\mathcal{K}$ denotes the matrix of operators with $m, n$ entry given by $\mathcal{K}_{m n}$, where

$$
\left(\mathcal{K}_{m n} \phi\right)(x)=\int_{0}^{1} k_{m n}(x, t) \phi(t) \mathrm{d} t \quad(0 \leqslant x \leqslant 1) .
$$

In the special case $w_{m}=w(m=1,2, \ldots, N),(2.1)$ corresponds to a system of equations with pure difference kernels. Weighted difference kernels with distinct $w_{m}$ were encountered recently by Biggs and Porter [2] in the context of wave-scattering theory. More generally, the integral equation

$$
\lambda \phi(x)=f(x)+\int_{a}^{b} k(x-t) z(t) \phi(t) \mathrm{d} t \quad(a \leqslant x \leqslant b),
$$

in which $z$ is piecewise constant, can be transformed into (2.1) by translating each subinterval on which $z$ is a fixed constant onto the unit interval. Chandler-Wilde et al. [4] recently considered the numerical treatment of an equation of the form (2.3) and an application to acoustic scattering.

In the problem considered by Biggs and Porter [2], a particular version of the system (2.1) arises in which $\lambda_{m}=0$ for $m=1,2, \ldots, N$,

$$
f_{m}(x) \equiv f_{\alpha}^{(m)}(x)=\mathrm{e}^{-\mathrm{i} \alpha w_{m} x} \quad(\alpha \in \mathbb{R}),
$$

and the kernels have the additional property $k_{m n}(x, t)=k_{n m}(t, x)$, corresponding to $\kappa_{m n}(x)=\kappa_{n m}(-x)$ in the present notation. Furthermore, $k_{m m}(x, t)=\kappa_{m m}\left(w_{m}(x-t)\right)$ is logarithmically singular for each $m$, all others kernels being continuous functions of both variables. Biggs and Porter [2] developed embedding formulae relating solutions of the system corresponding to different values of $\alpha$, which led to significant economy in the computational methods used to solve the scattering problem.

Here we seek to examine (2.1) from a different point of view and in greater generality. We suppose that $\kappa_{m n}$ is integrable for $m, n=1,2, \ldots, N$, that $f_{m} \in L_{2}(0,1)$ and that $\Lambda \neq 0$. These assumptions allow us to consider (2.2) via the equation

$$
\Lambda \phi=f+\mathcal{K} \phi \quad \text { in } \mathcal{H}
$$

where $\mathcal{H}=L_{2}\left((0,1), \mathbb{C}^{N}\right)$, taking $\Lambda \mathcal{I}-\mathcal{K}$ to be invertible. In fact, we shall shortly extend the investigation to a wider class of equations of which (2.5) may be regarded as the prototype. 
For the moment, however, we consider (2.5) and, as in Biggs and Porter [2], we make use of the $N \times N$ matrix operator $\mathcal{V}_{\alpha}$ with $m$ th diagonal entry given by

$$
\left(\mathcal{V}_{\alpha}^{(m)} \phi\right)(x)=w_{m} \int_{0}^{x} f_{\alpha}^{(m)}(x-t) \phi(t) \mathrm{d} t \quad(0 \leqslant x \leqslant 1),
$$

the kernel being defined by (2.4). With respect to the inner product

$$
(\phi, \psi)=\int_{0}^{1} \psi^{*}(x) \phi(x) \mathrm{d} x
$$

on $\mathcal{H}$, in which $\boldsymbol{\psi}^{*}=\overline{\boldsymbol{\psi}}^{\mathrm{T}}$, the adjoint operator $\mathcal{V}_{\alpha}^{*}$ satisfying $\left(\mathcal{V}_{\alpha} \boldsymbol{\phi}, \boldsymbol{\psi}\right)=\left(\boldsymbol{\phi}, \mathcal{V}_{\alpha}^{*} \boldsymbol{\psi}\right)$ is also diagonal. Its $m$ th diagonal entry is

$$
\left(\mathcal{V}_{\alpha}^{(m) *} \phi\right)(x)=w_{m} \int_{x}^{1} f_{\alpha}^{(m)}(x-t) \phi(t) \mathrm{d} t \quad(0 \leqslant x \leqslant 1),
$$

$\mathcal{V}_{\alpha}^{(m) *}$ being the adjoint of $\mathcal{V}_{\alpha}^{(m)}$ with respect to the scalar counterpart of (2.6). We note that $\mathcal{V}_{\alpha}$ and $\mathcal{V}_{\alpha}^{*}$ are related by

$$
\mathcal{U} \mathcal{V}_{\alpha}=\mathcal{V}_{\alpha}^{*} \mathcal{U}
$$

where the matrix operator $\mathcal{U}$ is defined by

$$
(\mathcal{U} \phi)(x)=\overline{\phi(1-x)}
$$

That is, $\mathcal{U}$ is the diagonal operator in which each diagonal component is the scalar 'reflection-conjugation' operator.

The vector $\mathcal{V}_{\alpha} \mathcal{K} \phi+\mathcal{K} \mathcal{V}_{\alpha}^{*} \phi$ is at the core of our approach. Its $m$ th component evaluated at $x \in[0,1]$ is

$$
\begin{aligned}
& \sum_{n=1}^{N} \int_{0}^{1} \phi_{n}(t) w_{m} \int_{0}^{x} \mathrm{e}^{-\mathrm{i} \alpha w_{m}(x-s)} \kappa_{m n}\left(w_{m} s-w_{n} t\right) \mathrm{d} s \mathrm{~d} t \\
& +\sum_{n=1}^{N} \int_{0}^{1} \phi_{n}(t) w_{n} \int_{0}^{t} \mathrm{e}^{\mathrm{i} \alpha w_{n}(t-s)} \kappa_{m n}\left(w_{m} x-w_{n} s\right) \mathrm{d} s \mathrm{~d} t \\
& =\sum_{n=1}^{N} \int_{0}^{1} \phi_{n}(t) \int_{-w_{n} t}^{w_{m} x} \mathrm{e}^{-\mathrm{i} \alpha\left(w_{m} x-w_{n} t-u\right)} \kappa_{m n}(u) \mathrm{d} u \mathrm{~d} t \\
& =\sum_{n=1}^{N} \int_{0}^{1} \phi_{n}(t)\left\{w_{n} \mathrm{e}^{-\mathrm{i} \alpha w_{m} x} \int_{0}^{t} \mathrm{e}^{\mathrm{i} \alpha w_{n}(t-s)} k_{m n}(0, s) \mathrm{d} s\right. \\
& \left.\quad+w_{m} \mathrm{e}^{\mathrm{i} \alpha w_{n} t} \int_{0}^{x} \mathrm{e}^{-\mathrm{i} \alpha w_{m}(x-s)} k_{m n}(s, 0) \mathrm{d} s\right\} \mathrm{d} t
\end{aligned}
$$

from which we deduce that

$$
\mathcal{V}_{\alpha} \mathcal{K} \boldsymbol{\phi}+\mathcal{K} \mathcal{V}_{\alpha}^{*} \phi=\sum_{n=1}^{N}\left\{\left(\phi, \mathcal{V}_{\alpha} \boldsymbol{T}^{(n)}\right) \boldsymbol{f}_{\alpha}^{(n)}+\left(\boldsymbol{\phi}, \boldsymbol{f}_{\alpha}^{(n)}\right) \mathcal{V}_{\alpha} \boldsymbol{S}^{(n)}\right\}
$$


in $\mathcal{H}$. Here we have introduced the vectors

$$
\boldsymbol{S}^{(n)}(x)=\left(k_{1 n}(x, 0), \ldots, k_{N n}(x, 0)\right)^{\mathrm{T}}, \quad \boldsymbol{T}^{(n)}(x)=\left(\overline{k_{n 1}(0, x)}, \ldots, \overline{k_{n N}(0, x)}\right)^{\mathrm{T}},
$$

together with

$$
\boldsymbol{f}_{\alpha}^{(n)}(x)=\left(0, \ldots, f_{\alpha}^{(n)}(x), \ldots, 0\right)^{\mathrm{T}},
$$

the non-zero element occurring in the $n$th component.

Using the identity

$$
\mathcal{V}_{\alpha} \phi+\mathcal{V}_{\alpha}^{*} \phi=\sum_{n=1}^{N} w_{n}\left(\boldsymbol{\phi}, \boldsymbol{f}_{\alpha}^{(n)}\right) \boldsymbol{f}_{\alpha}^{(n)} \quad(\boldsymbol{\phi} \in \mathcal{H}),
$$

we see that the operator $\mathcal{A} \equiv \Lambda \mathcal{I}-\mathcal{K}$ arising in (2.5) satisfies

$$
\mathcal{V}_{\alpha} \mathcal{A} \boldsymbol{\phi}+\mathcal{A} \mathcal{V}_{\alpha}^{*} \phi=\sum_{n=1}^{N}\left\{\left(\boldsymbol{\phi}, w_{n} \bar{\lambda}_{n} \boldsymbol{f}_{\alpha}^{(n)}-\mathcal{V}_{\alpha} \boldsymbol{T}^{(n)}\right) \boldsymbol{f}_{\alpha}^{(n)}-\left(\boldsymbol{\phi}, \boldsymbol{f}_{\alpha}^{(n)}\right) \mathcal{V}_{\alpha} \boldsymbol{S}^{(n)}\right\}
$$

Thus $\mathcal{V}_{\alpha} \mathcal{A}+\mathcal{A} \mathcal{V}_{\alpha}^{*}$ is an operator of rank $2 N$ and this is its key property.

A similar calculation gives the complementary formula

$$
\mathcal{V}_{\alpha}^{*} \mathcal{A} \phi+\mathcal{A} \mathcal{V}_{\alpha} \phi=\sum_{n=1}^{N}\left\{\left(\phi, w_{n} \bar{\lambda}_{n} \mathcal{U} \boldsymbol{f}_{\alpha}^{(n)}-\mathcal{V}_{\alpha}^{*} \boldsymbol{Q}^{(n)}\right) \mathcal{U} \boldsymbol{f}_{\alpha}^{(n)}-\left(\phi, \mathcal{U} \boldsymbol{f}_{\alpha}^{(n)}\right) \mathcal{V}_{\alpha}^{*} \boldsymbol{P}^{(n)}\right\}
$$

showing that $\mathcal{V}_{\alpha}^{*} \mathcal{A}+\mathcal{A} \mathcal{V}_{\alpha}$ also has rank $2 N$. The new quantities arising here are

$$
\boldsymbol{P}^{(n)}(x)=\left(k_{1 n}(x, 1), \ldots, k_{N n}(x, 1)\right)^{\mathrm{T}}, \quad \boldsymbol{Q}^{(n)}(x)=\left(\overline{k_{n 1}(1, x)}, \ldots, \overline{k_{n N}(1, x)}\right)^{\mathrm{T}} .
$$

The vectors $\boldsymbol{P}^{(j)}, \boldsymbol{Q}^{(j)}, \boldsymbol{S}^{(j)}$ and $\boldsymbol{T}^{(j)}$ are related through an integral identity which arises because their components are expressed in terms of the functions $\kappa_{m n}$. A direct calculation shows that

$$
w_{m}\left(\mathrm{e}^{-\mathrm{i} \alpha w_{n}} \boldsymbol{P}^{(n)}-\boldsymbol{S}^{(n)}, \boldsymbol{f}_{\alpha}^{(m)}\right)+w_{n}\left(\boldsymbol{f}_{\alpha}^{(n)}, \mathrm{e}^{-\mathrm{i} \alpha w_{m}} \boldsymbol{Q}^{(m)}-\boldsymbol{T}^{(m)}\right)=0,
$$

for $m, n=1,2, \ldots, N$.

Equations (2.11) and (2.12) are also related, since

$$
\left(\mathcal{V}_{\alpha}^{*} \mathcal{A}+\mathcal{A} \mathcal{V}_{\alpha}\right) \phi+\left(\mathcal{V}_{\alpha} \mathcal{A}+\mathcal{A} \mathcal{V}_{\alpha}^{*}\right) \phi=\sum_{n=1}^{N} w_{n}\left\{\left(\boldsymbol{\phi}, \mathcal{A}^{*} \boldsymbol{f}_{\alpha}^{(n)}\right) \boldsymbol{f}_{\alpha}^{(n)}+\left(\phi, \boldsymbol{f}_{\alpha}^{(n)}\right) \mathcal{A} \boldsymbol{f}_{\alpha}^{(n)}\right\}
$$

follows from (2.10). The adjoint of $\mathcal{A}$ is given by $\mathcal{A}^{*} \equiv \bar{\Lambda} \mathcal{I}-\mathcal{K}^{*}$ with $\mathcal{K}^{*}$, satisfying $(\mathcal{K} \boldsymbol{\phi}, \boldsymbol{\psi})=\left(\boldsymbol{\phi}, \mathcal{K}^{*} \boldsymbol{\psi}\right)$, having $m, n$ component $\mathcal{K}_{n m}^{*}$, where

$$
\left(\mathcal{K}_{n m}^{*} \phi\right)(x)=\int_{0}^{1} \overline{k_{n m}(t, x)} \phi(t) \mathrm{d} t \quad(0 \leqslant x \leqslant 1) .
$$


The connection (2.15) between (2.8) and (2.12) can be confirmed by evaluating $\mathcal{A} \boldsymbol{f}_{\alpha}^{(n)}$ and $\mathcal{A}^{*} \boldsymbol{f}_{\alpha}^{(n)}$ to give

$$
\left.\begin{array}{c}
w_{n} \mathcal{A} \boldsymbol{f}_{\alpha}^{(n)}=w_{n} \lambda_{n} \boldsymbol{f}_{\alpha}^{(n)}-\mathcal{V}_{\alpha} \boldsymbol{S}^{(n)}+\mathrm{e}^{-\mathrm{i} \alpha w_{n}} \mathcal{V}_{\alpha} \boldsymbol{P}^{(n)}-w_{n} \sum_{j=1}^{N}\left(\boldsymbol{f}_{\alpha}^{(n)}, \boldsymbol{T}^{(j)}\right) \boldsymbol{f}_{\alpha}^{(j)}, \\
w_{n} \mathcal{A}^{*} \boldsymbol{f}_{\alpha}^{(n)}=w_{n} \bar{\lambda}_{n} \boldsymbol{f}_{\alpha}^{(n)}-\mathcal{V}_{\alpha} \boldsymbol{T}^{(n)}+\mathrm{e}^{-\mathrm{i} \alpha w_{n}} \mathcal{V}_{\alpha} \boldsymbol{Q}^{(n)}-w_{n} \sum_{j=1}^{N}\left(\boldsymbol{f}_{\alpha}^{(n)}, \boldsymbol{S}^{(j)}\right) \boldsymbol{f}_{\alpha}^{(j)} \cdot
\end{array}\right\}
$$

The relationship (2.14) can be used with (2.10) and (2.16) to produce alternative forms of $\mathcal{A} \boldsymbol{f}_{\alpha}^{(n)}$ and $\mathcal{A}^{*} \boldsymbol{f}_{\alpha}^{(n)}$.

We now generalize the investigation by considering the class of bounded operators $\mathcal{A}$ in $\mathcal{H}$ for which $\mathcal{V}_{\alpha} \mathcal{A}+\mathcal{A} \mathcal{V}_{\alpha}^{*}$ is of finite rank, that is,

$$
\mathcal{V}_{\alpha} \mathcal{A} \phi+\mathcal{A V}_{\alpha}^{*} \phi=\sum_{j=1}^{M}\left(\phi, \boldsymbol{b}^{(j)}\right) \boldsymbol{a}^{(j)},
$$

for some given vectors

$$
\boldsymbol{a}^{(j)}=\left(a_{1}^{(j)}, a_{2}^{(j)}, \ldots, a_{M}^{(j)}\right)^{\mathrm{T}} \quad \text { and } \quad \boldsymbol{b}^{(j)}=\left(b_{1}^{(j)}, b_{2}^{(j)}, \ldots, b_{M}^{(j)}\right)^{\mathrm{T}}
$$

in $\mathcal{H}$ and some $M \in \mathbb{N}$. We suppress the dependence of $\boldsymbol{a}^{(j)}$ and $\boldsymbol{b}^{(j)}$ on $\alpha$, for clarity. It follows at once from (2.15) that if (2.17) holds, then

$$
\mathcal{V}_{\alpha}^{*} \mathcal{A} \phi+\mathcal{A} \mathcal{V}_{\alpha} \phi=\sum_{j=1}^{M^{\prime}}\left(\phi, \boldsymbol{d}^{(j)}\right) \boldsymbol{c}^{(j)},
$$

for some

$$
\boldsymbol{c}^{(j)}=\left(c_{1}^{(j)}, c_{2}^{(j)}, \ldots, c_{N}^{(j)}\right)^{\mathrm{T}} \quad \text { and } \quad \boldsymbol{d}^{(j)}=\left(d_{1}^{(j)}, d_{2}^{(j)}, \ldots, d_{N}^{(j)}\right)^{\mathrm{T}}
$$

in $\mathcal{H}$ (depending on $\alpha$ ) and some $M^{\prime} \in \mathbb{N}$ such that $M^{\prime} \leqslant 2 N+M$. Since the converse also holds, $\mathcal{A}$ satisfies (2.17) if and only if it satisfies (2.18).

If we now suppose that $\mathcal{A}$ is an invertible operator satisfying (2.17), then a simple rearrangement gives

$$
\mathcal{V}_{\alpha}^{*} \mathcal{A}^{-1} \phi+\mathcal{A}^{-1} \mathcal{V}_{\alpha} \phi=\sum_{j=1}^{M}\left(\boldsymbol{\phi}, \mathcal{A}^{*-1} \boldsymbol{b}^{(j)}\right) \mathcal{A}^{-1} \boldsymbol{a}^{(j)},
$$

which is of the form (2.18) and therefore $\mathcal{A}^{-1}$ also satisfies (2.17) for some vectors and some $M$. This observation forms the basis of the investigation and dictates the strategy we use in the following sections. We establish methods of reconstructing $\mathcal{A}$ from (2.17) and (2.18) which may then be applied to (2.19) or the equivalent equation expressed in the form (2.17). This process will lead to formulae for $\mathcal{A}^{-1}$ which require a knowledge of finitely many particular vectors, $\mathcal{A}^{-1} \boldsymbol{a}^{(j)}$ and $\mathcal{A}^{*-1} \boldsymbol{b}^{(j)}$ for $j=1,2, \ldots, M$ in the case of (2.19). 
From this description it would appear that the particular inverses needed to construct $\mathcal{A}^{-1}$ are fixed by the vectors occurring in the representation (2.17), but this is not the case as it is possible to transform the representation and thereby produce different forms for the inverse. We defer this aspect until later, however, as it is more direct to apply transformations when we have formulae available for the inverse.

\section{The construction of $\mathcal{A}$}

Our immediate objective is to determine $\mathcal{A}$ from both (2.17) and (2.18). A crucial ingredient in this step is the knowledge that the homogeneous versions of these equations have only the trivial solution, and this property is established first. We concentrate attention on (2.17) and infer the corresponding results for (2.18).

\subsection{The homogeneous case}

Suppose that $\mathcal{A}$ satisfies $\mathcal{V}_{\alpha} \mathcal{A}+\mathcal{A V}_{\alpha}^{*}=0$ and consider the general component equation

$$
\mathcal{V}_{\alpha}^{(m)} \mathcal{A}_{m n}+\mathcal{A}_{m n} \mathcal{V}_{\alpha}^{(n) *}=0
$$

where $\mathcal{A}_{m n}$ denotes the $m, n$ element of $\mathcal{A}$.

Let

$$
\left(\mathcal{E}_{\alpha}^{(m)} \phi\right)(x)=\mathrm{e}^{-\mathrm{i} \alpha w_{m} x} \phi(x),
$$

so that

$$
\left(\mathcal{E}_{\alpha}^{(m) *} \phi\right)(x)=\mathrm{e}^{\mathrm{i} \alpha w_{m} x} \phi(x),
$$

and thus

$$
\mathcal{E}_{\alpha}^{(m)} \mathcal{E}_{\alpha}^{(m) *}=\mathcal{I} \quad \text { and } \quad \mathcal{V}_{\alpha}^{(m)}=\mathcal{E}_{\alpha}^{(m)} \mathcal{V}_{m} \mathcal{E}_{\alpha}^{(m) *},
$$

where we have written $\mathcal{V}_{m}=\mathcal{V}_{0}^{(m)}$. Therefore, (3.1) can be expressed in the form

$$
\mathcal{E}_{\alpha}^{(m)}\left\{\mathcal{V}_{m}\left(\mathcal{E}_{\alpha}^{(m) *} \mathcal{A}_{m n} \mathcal{E}_{\alpha}^{(n)}\right)+\left(\mathcal{E}_{\alpha}^{(m) *} \mathcal{A}_{m n} \mathcal{E}_{\alpha}^{(n)}\right) \mathcal{V}_{n}^{*}\right\} \mathcal{E}_{\alpha}^{(n) *}=0
$$

whence $\mathcal{V}_{m} \mathcal{B}_{m n}+\mathcal{B}_{m n} \mathcal{V}_{n}^{*}=0$, where $\mathcal{B}_{m n}=\mathcal{E}_{\alpha}^{(m) *} \mathcal{A}_{m n} \mathcal{E}_{\alpha}^{(n)}$. Now $\mathcal{B}_{m n}=0$ implies $\mathcal{A}_{m n}=0$, since $\mathcal{E}_{\alpha}^{(m)}$ and $\mathcal{E}_{\alpha}^{(n) *}$ are invertible. It therefore suffices to establish the result in the case $\alpha=0$ and consider the simpler equation

$$
\mathcal{V}_{m} \mathcal{A}_{m n}+\mathcal{A}_{m n} \mathcal{V}_{n}^{*}=0
$$

in place of (3.1).

Suppose first that $\mathcal{A}_{m n}$ is generated by a continuous kernel $a_{m n}(x, t)$ having continuous first-order derivatives. Then (3.2) implies that

$$
w_{m} \int_{0}^{x} a_{m n}(s, t) \mathrm{d} s+w_{n} \int_{0}^{t} a_{m n}(x, s) \mathrm{d} s=0 \quad(0 \leqslant x, t \leqslant 1),
$$


and by differentiating this equation with respect to $x$ and $t$ we see that

$$
w_{n} \frac{\partial a_{m n}}{\partial x}+w_{m} \frac{\partial a_{m n}}{\partial t}=0 \quad(0 \leqslant x, t \leqslant 1), \quad a_{m n}(x, 0)=a_{m n}(0, x)=0 \quad(0 \leqslant x \leqslant 1) .
$$

It follows that $a_{m n}(x, t)=0$ in $[0,1] \times[0,1]$ and therefore $\mathcal{A}_{m n}=0$ in this case.

Now consider a general bounded operator $\mathcal{A}_{m n}$ satisfying (3.2), which implies that

$$
\mathcal{V}_{m}\left(\mathcal{V}_{m}^{p} \mathcal{A}_{m n} \mathcal{V}_{n}^{* p}\right)+\left(\mathcal{V}_{m}^{p} \mathcal{A}_{m n} \mathcal{V}_{n}^{* p}\right) \mathcal{V}_{n}^{*}=0
$$

for $p=0,1, \ldots$ Since $\mathcal{V}_{m} \mathcal{A}_{m n} \mathcal{V}_{n}^{*}$ is generated by an $L_{2}$ kernel, $\mathcal{V}_{m}^{3} \mathcal{A}_{m n} \mathcal{V}_{n}^{* 3}$ is generated by a continuous kernel with continuous first derivatives. Applying (3.3) with $m=n=3$ and appealing to the earlier result, we conclude that $\mathcal{V}_{m}^{3} \mathcal{A}_{m n} \mathcal{V}_{n}^{* 3}=0$. It follows that $\mathcal{A}_{m n}=0$ as $\mathcal{V}_{m}$ and $\mathcal{V}_{n}^{*}$ are injective. This establishes that if $\mathcal{A}$ is a bounded operator satisfying $\mathcal{V}_{\alpha} \mathcal{A}+\mathcal{A V}_{\alpha}^{*}=0$, then $\mathcal{A}=0$.

In a similar way, $\mathcal{V}_{\alpha}^{*} \mathcal{A}+\mathcal{A} \mathcal{V}_{\alpha}=0$ implies that $\mathcal{A}=0$.

\subsection{The inhomogeneous case}

In order to solve (2.17) in its inhomogeneous form, we shall make use of the matrix operator $\mathcal{P}$ with $m, n$ component defined by

$$
\left(\mathcal{P}_{m n} \phi\right)(x)= \begin{cases}w_{m} \phi\left(w_{m} x / w_{n}\right) & \text { for } 0 \leqslant x \leqslant \min \left(w_{n} / w_{m}, 1\right), \\ 0 & \text { otherwise. }\end{cases}
$$

It is easily verified that $\mathcal{P}=\mathcal{P}^{*}$.

Now the identity

$$
\begin{aligned}
& \int_{0}^{\min \left(w_{m} x / w_{n}, 1\right)} \mathrm{e}^{-\mathrm{i} \alpha\left(w_{m} x-w_{n} t\right)} \phi(t) \mathrm{d} t \\
& \quad+\int_{\min \left(w_{m} x / w_{n}, 1\right)}^{1} \mathrm{e}^{-\mathrm{i} \alpha\left(w_{m} x-w_{n} t\right)} \phi(t) \mathrm{d} t=\left(\phi, f_{\alpha}^{(n)}\right) f_{\alpha}^{(m)}(x)
\end{aligned}
$$

can be rearranged in the form

$$
\begin{aligned}
\frac{w_{m}}{w_{n}} \int_{0}^{\min \left(x, w_{n} / w_{m}\right)} & \mathrm{e}^{-\mathrm{i} \alpha w_{m}(x-t)} \phi\left(w_{m} t / w_{n}\right) \mathrm{d} t \\
& +\int_{\min \left(w_{m} x / w_{n}, 1\right)}^{1} \mathrm{e}^{-\mathrm{i} \alpha w_{n}\left(w_{m} x / w_{n}-t\right)} \phi(t) \mathrm{d} t=\left(\phi, f_{\alpha}^{(n)}\right) f_{\alpha}^{(m)}(x) .
\end{aligned}
$$

Expressed concisely, this is

$$
\mathcal{V}_{\alpha}^{(m)} \mathcal{P}_{m n} \phi+\mathcal{P}_{m n} \mathcal{V}_{\alpha}^{(n) *} \phi=w_{m} w_{n}\left(\phi, f_{\alpha}^{(n)}\right) f_{\alpha}^{(m)},
$$

and replacing $\phi$ by $\phi_{n}$ and summing over $n$ establishes the $m$ th component of

$$
\mathcal{V}_{\alpha} \mathcal{P} \phi+\mathcal{P} \mathcal{V}_{\alpha}^{*} \phi=\left(\phi, W \boldsymbol{f}_{\alpha}\right) W \boldsymbol{f}_{\alpha} \quad(\phi \in \mathcal{H})
$$

where the matrix $W=\operatorname{diag}\left(w_{1}, w_{2}, \ldots, w_{N}\right)$ and $\boldsymbol{f}_{\alpha}=\left(f_{\alpha}^{(1)}, f_{\alpha}^{(2)}, \ldots, f_{\alpha}^{(n)}\right)^{\mathrm{T}}$. 
Now consider (2.17), namely,

$$
\mathcal{V}_{\alpha} \mathcal{A}+\mathcal{A} \mathcal{V}_{\alpha}^{*}=\mathcal{F}, \quad \mathcal{F} \phi=\sum_{j=1}^{M}\left(\phi, b^{(j)}\right) \boldsymbol{a}^{(j)} .
$$

Then

$$
\mathcal{V}_{\alpha} \mathcal{F} \mathcal{V}_{\alpha}^{*} \phi=\sum_{j=1}^{M}\left(\phi, \mathcal{V}_{\alpha} \boldsymbol{b}^{(j)}\right) \mathcal{V}_{\alpha} \boldsymbol{a}^{(j)}=\sum_{j=1}^{M}\left(\phi, \mathcal{B}^{(j)} W \boldsymbol{f}_{\alpha}\right) \mathcal{A}^{(j)} W \boldsymbol{f}_{\alpha}
$$

where $\mathcal{A}^{(j)}$ and $\mathcal{B}^{(j)}$ are the diagonal operators on $\mathcal{H}$ whose $m$ th diagonal components are defined, respectively, by the convolutions

$$
\left(\mathcal{A}_{m}^{(j)} \phi\right)(x)=\int_{0}^{x} a_{m}^{(j)}(x-t) \phi(t) \mathrm{d} t, \quad\left(\mathcal{B}_{m}^{(j)} \phi\right)(x)=\int_{0}^{x} b_{m}^{(j)}(x-t) \phi(t) \mathrm{d} t
$$

for $j=1,2, \ldots, M$. We shall use the notation

$$
\mathcal{A}^{(j)} \phi=\boldsymbol{a}^{(j)} * \boldsymbol{\phi}, \quad \mathcal{B}^{(j)} \phi=\boldsymbol{b}^{(j)} * \boldsymbol{\phi}
$$

to represent the convolutions of vectors defined in this way and note in particular that $\mathcal{V}_{\alpha} \phi=\left(W \boldsymbol{f}_{\alpha}\right) * \boldsymbol{\phi}=\boldsymbol{\phi} *\left(W \boldsymbol{f}_{\alpha}\right)$.

Thus, employing (3.4) in (3.6), we have

$$
\mathcal{V}_{\alpha} \mathcal{F} \mathcal{V}_{\alpha}^{*} \phi=\sum_{j=1}^{M}\left(\mathcal{B}^{(j) *} \phi, W \boldsymbol{f}_{\alpha}\right) \mathcal{A}^{(j)} W \boldsymbol{f}_{\alpha}=\sum_{j=1}^{M}\left\{\mathcal{A}^{(j)} \mathcal{V}_{\alpha} \mathcal{P} \mathcal{B}^{(j) *} \phi+\mathcal{A}^{(j)} \mathcal{P} \mathcal{V}_{\alpha}^{*} \mathcal{B}^{(j) *} \phi\right\}
$$

and since $\mathcal{A}^{(j)}$ and $\mathcal{B}^{(j)}$ commute with $\mathcal{V}_{\alpha}$ (all being diagonal convolution operators), it follows that

$$
\mathcal{V}_{\alpha} \mathcal{F} \mathcal{V}_{\alpha}^{*}=\mathcal{V}_{\alpha}\left\{\sum_{j=1}^{M} \mathcal{A}^{(j)} \mathcal{P} \mathcal{B}^{(j) *}\right\}+\left\{\sum_{j=1}^{M} \mathcal{A}^{(j)} \mathcal{P} \mathcal{B}^{(j) *}\right\} \mathcal{V}_{\alpha}^{*}
$$

Hence, using (3.5) to replace $\mathcal{F}$,

$$
\mathcal{V}_{\alpha}\left\{\mathcal{V}_{\alpha} \mathcal{A} \mathcal{V}_{\alpha}^{*}-\sum_{j=1}^{M} \mathcal{A}^{(j)} \mathcal{P} \mathcal{B}^{(j) *}\right\}+\left\{\mathcal{V}_{\alpha} \mathcal{A} \mathcal{V}_{\alpha}^{*}-\sum_{j=1}^{M} \mathcal{A}^{(j)} \mathcal{P} \mathcal{B}^{(j) *}\right\} \mathcal{V}_{\alpha}^{*}=0
$$

and, as we have shown that the homogeneous equation of this form has only the trivial solution,

$$
\mathcal{V}_{\alpha} \mathcal{A} \mathcal{V}_{\alpha}^{*}=\sum_{j=1}^{M} \mathcal{A}^{(j)} \mathcal{P} \mathcal{B}^{(j) *}
$$

Conversely, if (3.8) holds, then

$$
\begin{aligned}
\mathcal{V}_{\alpha}\left\{\mathcal{V}_{\alpha} \mathcal{A} \mathcal{V}_{\alpha}^{*}\right\}+\left\{\mathcal{V}_{\alpha} \mathcal{A} \mathcal{V}_{\alpha}^{*}\right\} \mathcal{V}_{\alpha}^{*} & =\mathcal{V}_{\alpha}\left\{\sum_{j=1}^{M} \mathcal{A}^{(j)} \mathcal{P} \mathcal{B}^{(j) *}\right\}+\left\{\sum_{j=1}^{M} \mathcal{A}^{(j)} \mathcal{P} \mathcal{B}^{(j) *}\right\} \mathcal{V}_{\alpha}^{*} \\
& =\mathcal{V}_{\alpha} \mathcal{F} \mathcal{V}_{\alpha}^{*}
\end{aligned}
$$

by (3.7) and $\mathcal{V}_{\alpha} \mathcal{A}+\mathcal{A} \mathcal{V}_{\alpha}=\mathcal{F}$ follows. 
We have so far reduced (3.5) to (3.8) and the final step in obtaining a representation of $\mathcal{A}$ requires the removal of the operators $\mathcal{V}_{\alpha}$ and $\mathcal{V}_{\alpha}^{*}$ from the left-hand side of the latter equation. This is immediate if there exist bounded operators $\tilde{\mathcal{A}}^{(j)}$ and $\tilde{\mathcal{B}}^{(j)}$ such that

$$
\mathcal{A}^{(j)}=\mathcal{V}_{\alpha} \tilde{\mathcal{A}}^{(j)}, \quad \mathcal{B}^{(j)}=\mathcal{V}_{\alpha} \tilde{\mathcal{B}}^{(j)} \quad(j=1,2, \ldots, M),
$$

for then (3.8) can be written in the form

$$
\mathcal{V}_{\alpha}\left\{\mathcal{A}-\sum_{j=1}^{M} \tilde{\mathcal{A}}^{(j)} \mathcal{P} \tilde{\mathcal{B}}^{(j)}\right\} \mathcal{V}_{\alpha}^{*}=0
$$

from which we deduce that

$$
\mathcal{A}=\sum_{j=1}^{M} \tilde{\mathcal{A}}^{(j)} \mathcal{P} \tilde{\mathcal{B}}^{(j)} .
$$

The converse, that (3.9) and (3.10) together imply (3.8), is trivial. We note that $\tilde{\mathcal{A}}^{(j)}$ and $\tilde{\mathcal{B}}^{(j)}$ are diagonal operators which therefore commute with $\mathcal{V}_{\alpha}$. The case in which operators $\tilde{\mathcal{A}}^{(j)}$ and $\tilde{\mathcal{B}}^{(j)}$ satisfying (3.9) do not exist and (3.8) has to be solved for $\mathcal{A}$ less directly will not be pursued.

To give a concrete form to these expressions we first evaluate the $m$ th component of the vector $\mathcal{A}^{(j)} \mathcal{P} \mathcal{B}^{(j) *} \phi$ and obtain

$$
\begin{aligned}
& \left(\mathcal{V}_{\alpha} \mathcal{A} \mathcal{V}_{\alpha}^{*} \phi\right)_{m}(x) \\
& \quad=\sum_{n=1}^{N} w_{m} w_{n} \int_{0}^{\min \left(x / w_{n}, 1 / w_{m}\right)} a_{m}^{(j)}\left(x-w_{n} s\right) \mathrm{d} s \int_{w_{m} s}^{1} \overline{b_{n}^{(j)}\left(t-w_{m} s\right)} \phi_{n}(t) \mathrm{d} t \\
& \quad=\sum_{n=1}^{N} w_{m} w_{n} \int_{0}^{1} \phi_{n}(t) \mathrm{d} t \int_{0}^{\ell(x, t)} a_{m}^{(j)}\left(x-w_{n} s\right) \overline{b_{n}^{(j)}\left(t-w_{m} s\right)} \mathrm{d} s,
\end{aligned}
$$

where $\ell(x, t)=\min \left(x / w_{n}, t / w_{m}\right)$, for almost all $x \in[0,1]$, the second version following from reversal of the integration order in the first.

Before making the corresponding calculation for $\mathcal{A} \phi$ using (3.10), we have to examine the forms of $\tilde{\mathcal{A}}^{(j)}$ and $\tilde{\mathcal{B}}^{(j)}$. For the bounded operator $\tilde{\mathcal{A}}^{(j)}$ to exist satisfying $\mathcal{A}^{(j)}=\mathcal{V}_{\alpha} \tilde{\mathcal{A}}^{(j)}$, it is sufficient that $\boldsymbol{a}^{(j)}$ can be written in the form

$$
\boldsymbol{a}^{(j)}=\Delta_{j} \boldsymbol{f}_{\alpha}+\mathcal{V}_{\alpha} \boldsymbol{a}^{(j)},
$$

for some constant diagonal matrix $\Delta_{j}$ and some vector $\mathbf{a}^{(j)} \in \mathcal{H}$. This representation of $\boldsymbol{a}^{(j)}$ gives

$$
\mathcal{A}^{(j)} \phi=\boldsymbol{a}^{(j)} * \boldsymbol{\phi}=\Delta_{j} \boldsymbol{f}_{\alpha} * \boldsymbol{\phi}+\left(\mathcal{V}_{\alpha} \mathbf{a}^{(j)}\right) * \boldsymbol{\phi}=\mathcal{V}_{\alpha}\left\{W^{-1} \Delta_{j} \phi+\boldsymbol{a}^{(j)} * \boldsymbol{\phi}\right\},
$$

which is of the desired form, on recalling that $\mathcal{V}_{\alpha} \boldsymbol{\phi}=W \boldsymbol{f}_{\alpha} * \boldsymbol{\phi}$ and verifying that $\left(\mathcal{V}_{\alpha} \mathbf{a}^{(j)}\right) * \boldsymbol{\phi}=\mathcal{V}_{\alpha}\left(\mathbf{a}^{(j)} * \boldsymbol{\phi}\right)$. 
The structure (3.11) holds in particular if the components of $\boldsymbol{a}^{(j)}$ are continuous in $[0,1]$ and have derivatives in $L_{2}(0,1)$, in which case we can take

$$
\left.\begin{array}{c}
\tilde{\mathcal{A}}^{(j)} \phi=W^{-1} \Delta_{j} \phi+\boldsymbol{a}^{(j)} * \boldsymbol{\phi}, \quad \boldsymbol{a}^{(j)}=W^{-1} \boldsymbol{a}^{(j)^{\prime}}+\mathrm{i} \alpha \boldsymbol{a}^{(j)}, \\
\Delta_{j}=\operatorname{diag}\left(a_{1}^{(j)}(0), a_{2}^{(j)}(0), \ldots, a_{M}^{(j)}(0)\right) .
\end{array}\right\}
$$

Assuming that $\boldsymbol{b}^{(j)}$ satisfies the same hypotheses as $\boldsymbol{a}^{(j)}$ and that $\tilde{\mathcal{B}}^{(j)}$ therefore has the corresponding representation to that in (3.12), we can evaluate the explicit version of $\mathcal{A}$ given by (3.10). After some manipulation, we find that the $m$ th component of the vector $\mathcal{A} \phi$ is given by

$$
(\mathcal{A} \phi)_{m}(x)=\sum_{n=1}^{N}\left\{e_{m n}\left(\mathcal{P}_{m n} \phi_{n}\right)(x)+\int_{0}^{1} r_{m n}(x, t) \phi_{n}(t) \mathrm{d} t\right\}
$$

almost everywhere in $[0,1]$, where

$$
e_{m n}=\left(w_{m} w_{n}\right)^{-1} \sum_{j=1}^{M} a_{m}^{(j)}(0) \overline{b_{n}^{(j)}(0)}
$$

and

$$
\begin{aligned}
& r_{m n}(x, t)=\sum_{j=1}^{M} w_{m} w_{n} \int_{0}^{\ell(x, t)} a_{m}^{(j)}\left(x-w_{n} s\right) \overline{b_{n}^{(j)}\left(t-w_{m} s\right)} \mathrm{d} s \\
& +\sum_{j=1}^{M} \begin{cases}a_{m}^{(j)}(0) \overline{b_{n}^{(j)}\left(t-w_{m} x / w_{n}\right)} & \left(w_{m} x<w_{n} t\right), \\
\overline{b_{n}^{(j)}(0)} a_{m}^{(j)}\left(x-w_{n} t / w_{m}\right) & \left(w_{m} x>w_{n} t\right),\end{cases}
\end{aligned}
$$

for almost all $x, t \in[0,1] \times[0,1]$. An alternative, more-succinct form for the kernels that may be deduced from (3.14) avoids the need to form the components of $\boldsymbol{a}^{(j)}$ and $\boldsymbol{b}^{(j)}$. This is

$$
r_{m n}(x, t)=\left(\frac{\partial}{\partial x}+\mathrm{i} \alpha w_{m}\right)\left(\frac{\partial}{\partial t}-\mathrm{i} \alpha w_{n}\right) \sum_{j=1}^{M} \int_{0}^{\ell(x, t)} a_{m}^{(j)}\left(x-w_{n} s\right) \overline{b_{n}^{(j)}\left(t-w_{m} s\right)} \mathrm{d} s,
$$

also for almost all $x, t \in[0,1] \times[0,1]$. We have therefore solved (2.17) for $\mathcal{A}$ and have identified the class of operators that satisfy (2.17), subject to certain hypotheses. In particular, it can be verified that the application of (3.13) to (2.11) recovers the particular operator $\mathcal{A}=\Lambda \mathcal{I}-\mathcal{K}$ arising from the integral-equation system (2.1). However, the class of operators $\mathcal{A}$ satisfying (2.17) includes those given by (3.13) for any $\boldsymbol{a}^{(j)}$ and $\boldsymbol{b}^{(j)}$ that are continuous in $[0,1]$ and have derivatives in $L_{2}(0,1)$. This observation makes it clear that we are dealing with much more general operators than those generated by weighted difference kernels.

We now deal with (2.18), that is,

$$
\mathcal{V}_{\alpha}^{*} \mathcal{A}+\mathcal{A} \mathcal{V}_{\alpha}=\mathcal{F}^{\prime}, \quad \mathcal{F}^{\prime} \phi=\sum_{j=1}^{M^{\prime}}\left(\boldsymbol{\phi}, \boldsymbol{d}^{(j)}\right) \boldsymbol{c}^{(j)}
$$


by employing the following strategy. With the aid of (2.7) we write (3.16) as

$$
\mathcal{V}_{\alpha}(\mathcal{U} \mathcal{A} \mathcal{U}) \phi+(\mathcal{U} \mathcal{A} \mathcal{U}) \mathcal{V}_{\alpha}^{*} \phi=\sum_{j=1}^{M^{\prime}}\left(\phi, \mathcal{U} \boldsymbol{d}^{(j)}\right) \mathcal{U} \boldsymbol{c}^{(j)}
$$

which has the form of (2.17). By identifying $\mathcal{U} \mathcal{A U}, \mathcal{U} \boldsymbol{c}^{(j)}, \mathcal{U} \boldsymbol{d}^{(j)}$ and $M^{\prime}$ with $\mathcal{A}, \boldsymbol{a}^{(j)}$, $\boldsymbol{b}^{(j)}$ and $M$, respectively, we directly obtain parallels of the results obtained for (2.17). We deduce that

$$
\left.\begin{array}{c}
\mathcal{V}_{\alpha}^{*} \mathcal{A} \phi+\mathcal{A} \mathcal{V}_{\alpha} \phi=\sum_{j=1}^{M^{\prime}}\left(\boldsymbol{\phi}, \boldsymbol{d}^{(j)}\right) \boldsymbol{c}^{(j)} \quad \Longleftrightarrow \quad \mathcal{V}_{\alpha}^{*} \mathcal{A} \mathcal{V}_{\alpha}=\sum_{j=1}^{M^{\prime}} \mathcal{C}^{(j) *} \mathcal{U} \mathcal{P} \mathcal{D}^{(j)}, \\
C^{(j)} \phi=\left(\mathcal{U} \boldsymbol{c}^{(j)}\right) * \boldsymbol{\phi}, \quad D^{(j)} \phi=\left(\mathcal{U} \boldsymbol{d}^{(j)}\right) * \boldsymbol{\phi} .
\end{array}\right\}
$$

Here we have made use of (2.7) again, together with the properties

$$
\mathcal{U} \mathcal{C}^{(j)}=\mathcal{C}^{(j) *} \mathcal{U} \text { and } \mathcal{U D}^{(j)}=\mathcal{D}^{(j) *} \mathcal{U}
$$

If there are bounded (diagonal) operators $\tilde{\mathcal{C}}^{(j)}$ and $\tilde{\mathcal{D}}^{(j)}$ satisfying

$$
\mathcal{C}^{(j)}=\mathcal{V}_{\alpha} \tilde{\mathcal{C}}^{(j)}=\tilde{\mathcal{C}}^{(j)} \mathcal{V}_{\alpha}, \quad \mathcal{D}^{(j)}=\mathcal{V}_{\alpha} \tilde{\mathcal{D}}^{(j)}=\tilde{\mathcal{D}}^{(j)} \mathcal{V}_{\alpha}
$$

for $j=1,2, \ldots, N$, we obtain an explicit expression for the solution $\mathcal{A}$ of (2.18) in the form

$$
\mathcal{A}=\sum_{j=1}^{M^{\prime}} \tilde{\mathcal{C}}^{(j) *} \mathcal{U} \mathcal{P} \mathcal{U} \tilde{\mathcal{D}}^{(j)}
$$

Otherwise, $\mathcal{A}$ has to be extricated from (3.18) less directly.

The counterpart of (3.13) for (2.18) requires that the components of $\boldsymbol{c}^{(j)}$ and $\boldsymbol{d}^{(j)}$ are continuous in $[0,1]$ and have derivatives in $L_{2}(0,1)$. In particular, the form corresponding to $(3.11)$ is

$$
\boldsymbol{c}^{(j)}=\Delta_{j}^{\prime} \boldsymbol{f}_{\alpha}+\mathcal{V}_{\alpha}^{*} \boldsymbol{c}^{(j)}
$$

for some constant diagonal matrix $\Delta_{j}^{\prime}$ and some vector $\boldsymbol{c}^{(j)} \in \mathcal{H}$, and similarly for $\boldsymbol{d}^{(j)}$. Under these conditions, applying (3.13) to (3.17) we find that (2.18) implies

$$
(\mathcal{A} \phi)_{m}(x)=\sum_{n=1}^{N}\left\{e_{m n}^{\prime}\left(\mathcal{U} \mathcal{P}_{m n} \mathcal{U} \phi_{n}\right)(x)+\int_{0}^{1} r_{m n}^{\prime}(x, t) \phi_{n}(t) \mathrm{d} t\right\}
$$

almost everywhere in $[0,1]$, where

$$
e_{m n}^{\prime}=\left(w_{m} w_{n}\right)^{-1} \sum_{j=1}^{M^{\prime}} c_{m}^{(j)}(1) \overline{d_{n}^{(j)}(1)}
$$

and

$$
r_{m n}^{\prime}(x, t)=\left(\frac{\partial}{\partial x}+\mathrm{i} \alpha w_{m}\right)\left(\frac{\partial}{\partial t}-\mathrm{i} \alpha w_{n}\right) \sum_{j=1}^{M^{\prime}} \int_{0}^{\ell(1-x, 1-t)} c_{m}^{(j)}\left(x+w_{n} s\right) \overline{d_{n}^{(j)}\left(t+w_{m} s\right)} \mathrm{d} s
$$

(where $\left.\ell(x, t)=\min \left(x / w_{n}, t / w_{m}\right)\right)$ for almost all $x, t \in[0,1] \times[0,1]$. Term-by-term differentiation leads to an alternative form for $r^{\prime}(x, t)$ that parallels (3.14). 


\section{The determination of $\mathcal{A}^{-1}$}

We now apply the results of the preceding section to the determination of $\mathcal{A}^{-1}$. It is convenient to retain the earlier notation as far as possible and this can be achieved simply by setting aside the connection (2.15) between (2.17) and (2.18) and redefining $\boldsymbol{c}^{(j)}$ and $\boldsymbol{d}^{(j)}$ as follows.

Suppose that $\mathcal{A} \phi=f$ in $\mathcal{H}$, where $\mathcal{A}$ satisfies (2.17) and is invertible. Then

$$
\mathcal{V}_{\alpha}^{*} \mathcal{A}^{-1} \boldsymbol{f}+\mathcal{A}^{-1} \mathcal{V}_{\alpha} \boldsymbol{f}=\mathcal{F}^{\prime} \boldsymbol{f} \equiv \sum_{j=1}^{M}\left(\boldsymbol{f}, \boldsymbol{d}^{(j)}\right) \boldsymbol{c}^{(j)},
$$

as anticipated in (2.19), where $\boldsymbol{c}^{(j)}$ and $\boldsymbol{d}^{(j)}$ are now defined by

$$
\mathcal{A} \boldsymbol{c}^{(j)}=\boldsymbol{a}^{(j)}, \quad \mathcal{A}^{*} \boldsymbol{d}^{(j)}=\boldsymbol{b}^{(j)} \quad(j=1,2, \ldots, M) .
$$

As (4.1) is of the form (2.18), we may deduce $\mathcal{A}^{-1} \boldsymbol{f}$ by identifying $M^{\prime}$ with $M$ and using the derivation given in $\S 3$.

In particular, if the vectors $\boldsymbol{c}^{(j)}$ and $\boldsymbol{d}^{(j)}$ that satisfy (4.2) are continuous in $[0,1]$ and have derivatives in $L_{2}(0,1)$, then the formula (3.20) with $\mathcal{A}^{-1} \boldsymbol{f}$ replacing $\mathcal{A} \boldsymbol{\phi}$ gives an explicit expression for the components of $\mathcal{A}^{-1} \boldsymbol{f}$. If $\mathcal{A}=\Lambda \mathcal{I}-\mathcal{K}$, where $\mathcal{K}$ is a compact operator, as envisaged in $\S 2$, we can be sure that (3.21) simplifies to give $\mathcal{A}^{-1}=\Lambda^{-1} \mathcal{I}+\mathcal{R}$, where $\mathcal{R}$ is also compact. In this case,

$$
\left(\mathcal{A}^{-1} \boldsymbol{f}\right)_{m}(x)=\lambda_{m}^{-1} f_{m}(x)+\sum_{n=1}^{N} \int_{0}^{1} r_{m n}^{\prime}(x, t) f_{n}(t) \mathrm{d} t,
$$

for almost all $x \in[0,1], r_{m n}^{\prime}$ being given by (3.22) with $M^{\prime}$ replaced by $M$ and with $\boldsymbol{c}^{(j)}$ and $\boldsymbol{d}^{(j)}$ defined by (4.2). If the solutions of (4.2) are such that the formula (3.20) cannot be applied, we have to return to (3.18) with $\mathcal{A}$ replaced by $\mathcal{A}^{-1}$ and extract the latter less directly.

The direct approach to the determination of $\mathcal{A}^{-1} \boldsymbol{f}$ is therefore to solve the $2 M$ equations (4.2) for $\boldsymbol{c}^{(j)}$ and $\boldsymbol{d}^{(j)}$. We note that these vectors satisfy the reciprocal relations

$$
\left(\boldsymbol{b}^{(j)}, \boldsymbol{c}^{(i)}\right)=\left(\boldsymbol{d}^{(j)}, \boldsymbol{a}^{(i)}\right), \quad i, j=1,2, \ldots, M .
$$

\subsection{Transformations of $c^{(j)}$ and $d^{(j)}$}

There are, however, alternative versions of $\mathcal{A}^{-1} \boldsymbol{f}$ in which some or all of $\boldsymbol{c}^{(j)}$ and $\boldsymbol{d}^{(j)}$ are replaced by other vectors that may be more directly relevant to a particular application or preferable in some other way. Vectors of the form (3.19) that admit the representation (4.3) are desirable, for example. We now investigate how different versions of $\mathcal{F}^{\prime}$ and therefore of $\mathcal{A}^{-1}$ may be constructed.

There may seem to be little scope for creating significantly different representations of $\mathcal{F}^{\prime}$, as $\mathcal{F}^{\prime} \boldsymbol{f}$ is in the fixed subspace of $\mathcal{H}$ spanned by $\boldsymbol{c}^{(1)}, \ldots, \boldsymbol{c}^{(M)}$ and, similarly, $\mathcal{F}^{\prime *} \boldsymbol{f}$ 
is in the subspace spanned by $\boldsymbol{d}^{(1)}, \ldots, \boldsymbol{d}^{(M)}$. This impression is misleading, however, as every vector in $\mathcal{H}$ can be mapped onto these subspaces by using (4.1) and its adjoint

$$
\mathcal{V}_{\alpha}^{*} \mathcal{A}^{*-1} \boldsymbol{f}+\mathcal{A}^{*-1} \mathcal{V}_{\alpha} \boldsymbol{f}=\mathcal{F}^{*} \boldsymbol{f} \equiv \sum_{j=1}^{M}\left(\boldsymbol{f}, \boldsymbol{c}^{(j)}\right) \boldsymbol{d}^{(j)} .
$$

We may therefore generate transformations in the following way.

Suppose that we seek to replace $\boldsymbol{c}^{(j)}$ and $\boldsymbol{d}^{(j)}$ for $j=1,2, \ldots, M$. We choose any vectors $\boldsymbol{g}^{(j)}$ and $\boldsymbol{h}^{(j)}$ in $\mathcal{H}$ for $j=1,2, \ldots, M$ and define the replacements by

$$
\left.\begin{array}{l}
\hat{\boldsymbol{c}}^{(j)}=\mathcal{V}_{\alpha}^{*} \mathcal{A}^{-1} \boldsymbol{g}^{(j)}+\mathcal{A}^{-1} \mathcal{V}_{\alpha} \boldsymbol{g}^{(j)} \\
\hat{\boldsymbol{d}}^{(j)}=\mathcal{V}_{\alpha}^{*} \mathcal{A}^{*-1} \boldsymbol{h}^{(j)}+\mathcal{A}^{*-1} \mathcal{V}_{\alpha} \boldsymbol{h}^{(j)}
\end{array}\right\} \quad(j=1,2, \ldots, M)
$$

These are linear combinations of the vectors $\boldsymbol{c}^{(j)}$ and $\boldsymbol{d}^{(j)}$, respectively, by virtue of (4.1) and (4.4), namely

$$
\hat{\boldsymbol{c}}^{(i)}=\sum_{j=1}^{M}\left(\boldsymbol{g}^{(i)}, \boldsymbol{d}^{(j)}\right) \boldsymbol{c}^{(j)}, \quad \hat{\boldsymbol{d}}^{(i)}=\sum_{j=1}^{M}\left(\boldsymbol{h}^{(i)}, \boldsymbol{c}^{(j)}\right) \boldsymbol{d}^{(j)} \quad(i=1,2, \ldots, M) .
$$

The relationships between the vectors $\boldsymbol{c}^{(j)}$ and $\boldsymbol{d}^{(j)}$ and their replacements can therefore be expressed in the concise forms

$$
\left(\boldsymbol{c}^{(1)}, \ldots, \boldsymbol{c}^{(M)}\right) G=\left(\hat{\boldsymbol{c}}^{(1)}, \ldots, \hat{\boldsymbol{c}}^{(M)}\right), \quad\left(\boldsymbol{d}^{(1)}, \ldots, \boldsymbol{d}^{(M)}\right) H=\left(\hat{\boldsymbol{d}}^{(1)}, \ldots, \hat{\boldsymbol{d}}^{(M)}\right),
$$

where $G$ and $H$ are the $M \times M$ matrices having $i, j$ components $\left(\boldsymbol{g}^{(j)}, \boldsymbol{d}^{(i)}\right)$ and $\left(\boldsymbol{h}^{(j)}, \boldsymbol{c}^{(i)}\right)$, respectively. We will assume that the vectors $\boldsymbol{g}^{(j)}$ and $\boldsymbol{h}^{(j)}$ are chosen so that $G$ and $H$ are non-singular.

Moreover, (4.6) implies that

$$
\left(\boldsymbol{h}^{(k)}, \hat{\boldsymbol{c}}^{(i)}\right)=\overline{\left(\boldsymbol{g}^{(i)}, \hat{\boldsymbol{d}}^{(k)}\right)}=\sum_{j=1}^{M} \overline{\left(\boldsymbol{g}^{(i)}, \boldsymbol{d}^{(j)}\right)}\left(\boldsymbol{h}^{(k)}, \boldsymbol{c}^{(j)}\right),
$$

the first equality being a reciprocal principle for (4.5), and hence

$$
\hat{H}=\hat{G}^{*}=G^{*} H
$$

where $\hat{G}$ and $\hat{H}$ are $M \times M$ matrices with $i, j$ components $\left(\boldsymbol{g}^{(j)}, \hat{\boldsymbol{d}}^{(i)}\right)$ and $\left(\boldsymbol{h}^{(j)}, \hat{\boldsymbol{c}}^{(i)}\right)$, respectively. Since

$$
\left(\left(\boldsymbol{f}, \hat{\boldsymbol{d}}^{(1)}\right), \ldots,\left(\boldsymbol{f}, \hat{\boldsymbol{d}}^{(M)}\right)\right)^{\mathrm{T}}=H^{*}\left(\left(\boldsymbol{f}, \boldsymbol{d}^{(1)}\right), \ldots,\left(\boldsymbol{f}, \boldsymbol{d}^{(M)}\right)\right)^{\mathrm{T}}
$$

follows from (4.7), and

$$
\mathcal{F}^{\prime} \boldsymbol{f}=\left(\boldsymbol{c}^{(1)}, \ldots, \boldsymbol{c}^{(M)}\right)\left(\left(\boldsymbol{f}, \boldsymbol{d}^{(1)}\right), \ldots,\left(\boldsymbol{f}, \boldsymbol{d}^{(M)}\right)\right)^{\mathrm{T}},
$$


we have

$$
\mathcal{F}^{\prime} \boldsymbol{f}=\left(\hat{\boldsymbol{c}}^{(1)}, \ldots, \hat{\boldsymbol{c}}^{(M)}\right) \hat{G}^{-1}\left(\left(\boldsymbol{f}, \hat{\boldsymbol{d}}^{(1)}\right), \ldots,\left(\boldsymbol{f}, \hat{\boldsymbol{d}}^{(M)}\right)\right)^{\mathrm{T}} .
$$

We note that $\hat{G}$ is determined by the transformed vectors $\hat{\boldsymbol{c}}^{(j)}$ and $\hat{\boldsymbol{d}}^{(j)}$ together with the generating functions $\boldsymbol{g}^{(j)}$ and $\boldsymbol{h}^{(j)}$, and the revised form (4.9) of $\mathcal{F}^{\prime}$ is therefore independent of the original vectors $\boldsymbol{c}^{(j)}$ and $\boldsymbol{d}^{(j)}$. The structure of (4.1) is restored in (4.9) by absorbing $\hat{G}^{-1}$ into either the matrix $\left(\hat{\boldsymbol{c}}^{(1)}, \ldots, \hat{\boldsymbol{c}}^{(M)}\right)$ or the vector

$$
\left(\left(\boldsymbol{f}, \hat{\boldsymbol{d}}^{(1)}\right), \ldots,\left(\boldsymbol{f}, \hat{\boldsymbol{d}}^{(M)}\right)\right)^{\mathrm{T}} .
$$

Thus it is clear that $\mathcal{A}^{-1} \boldsymbol{f}$ is expressible entirely in terms of the new vectors $\hat{\boldsymbol{c}}^{(j)}$ and $\hat{\boldsymbol{d}}^{(j)}$. As these are formed from the $4 M$ components $\mathcal{A}^{-1} \boldsymbol{g}^{(j)}, \mathcal{A}^{-1} \mathcal{V}_{\alpha} \boldsymbol{g}^{(j)}, \mathcal{A}^{*-1} \boldsymbol{h}^{(j)}$ and $\mathcal{A}^{*-1} \mathcal{V}_{\alpha} \boldsymbol{h}^{(j)}$ for $j=1,2, \ldots, M$, compared with the $2 M$ vectors needed by the direct approach, this general transformation is not attractive, however. The requirement reduces if $\mathcal{V}_{\alpha} \boldsymbol{g}^{(j)}$ can be expressed in terms of $\boldsymbol{g}^{(1)}, \ldots, \boldsymbol{g}^{(M)}$ for each $j$, and similarly for each $\mathcal{V}_{\alpha} \boldsymbol{h}^{(j)}$. There are several choices of $\boldsymbol{g}^{(j)}$ and $\boldsymbol{h}^{(j)}$ for which this situation arises and an illustration is given of one particular example shortly.

Variants of the transformation given may be formulated in a similar way. For example, if it is convenient to retain some of the original vectors $\boldsymbol{c}^{(j)}$ and $\boldsymbol{d}^{(j)}$, the transformation matrices in (4.8) can be expressed in block form, with identity and zero matrices occupying appropriate blocks. Furthermore, vectors requiring fewer than the $4 M$ inverses needed for the general transformation or those having the structure (3.19) may be sought. We remark that if, for a given $\mathcal{A}$, particular vectors $\hat{\boldsymbol{c}}^{(j)}$ and $\hat{\boldsymbol{d}}^{(j)}$ satisfying (3.19) can be found for $j=1,2, \ldots, M$, then the vectors in all other representations of $\mathcal{F}^{\prime}$ must be continuous in $[0,1]$ and have derivatives in $L_{2}(0,1)$, through the transformation process.

Rather than attempting to express these variations of the basic transformation in general terms, we return to the integral-equation system of $\S 2$ which allows us to give explicit illustrations.

\section{The integral-equation system}

The system (2.1) leads to (2.11), which may be aligned with the notation in (2.17) by taking $M=2 N$ and

$$
\left.\begin{array}{ll}
\boldsymbol{a}^{(j)}=\boldsymbol{f}_{\alpha}^{(j)}, & \boldsymbol{a}^{(N+j)}=-\mathcal{V}_{\alpha} \boldsymbol{S}^{(j)} \\
\boldsymbol{b}^{(j)}=w_{j} \bar{\lambda}_{j} \boldsymbol{f}_{\alpha}^{(j)}-\mathcal{V}_{\alpha} \boldsymbol{T}^{(j)}, & \boldsymbol{b}^{(N+j)}=\boldsymbol{f}_{\alpha}^{(j)}
\end{array}\right\} \quad(j=1,2, \ldots, N)
$$

The direct approach to forming the solution $\boldsymbol{\phi}=(\Lambda \mathcal{I}-\mathcal{K})^{-1} \boldsymbol{f}$ of $(2.2)$ therefore requires the determination of the $4 N$ vectors

$$
\left.\begin{array}{rlrl}
\boldsymbol{c}^{(j)} & =\mathcal{A}^{-1} \boldsymbol{f}_{\alpha}^{(j)}, & \boldsymbol{c}^{(N+j)} & =-\mathcal{A}^{-1} \mathcal{V}_{\alpha} \boldsymbol{S}^{(j)} \\
\boldsymbol{d}^{(j)} & =w_{j} \bar{\lambda}_{j} \mathcal{A}^{*-1} \boldsymbol{f}_{\alpha}^{(j)}-\mathcal{A}^{*-1} \mathcal{V}_{\alpha} \boldsymbol{T}^{(j)}, & \boldsymbol{d}^{(N+j)} & =\mathcal{A}^{*-1} \boldsymbol{f}_{\alpha}^{(j)}
\end{array}\right\} \quad(j=1,2, \ldots, N)
$$

Two alternative transformation strategies are suggested by this structure. 
One is to express $\mathcal{F}^{\prime}$ and hence $\mathcal{A}^{-1}$ entirely in terms of solutions of

$$
\mathcal{A} \phi_{\alpha}^{(j)}=\boldsymbol{f}_{\alpha}^{(j)}, \quad \mathcal{A}^{*} \boldsymbol{\psi}_{\alpha}^{(j)}=\boldsymbol{f}_{\alpha}^{(j)} \quad(j=1,2, \ldots, N) .
$$

The motivation for this approach is that $2 N$ of the vectors needed to construct $\mathcal{A}^{-1}$ are already of the required form. Some saving is therefore possible compared with the general case, as we can retain $\boldsymbol{c}^{(j)}$ and $\boldsymbol{d}^{(N+j)}$ for $j=1,2, \ldots, N$ and replace only the remaining members of the two sets defining $\mathcal{F}^{\prime}$. The second transformation is complementary to this, in the sense that we can retain $\boldsymbol{c}^{(N+j)}$ and $\boldsymbol{d}^{(j)}$, and replace $\boldsymbol{c}^{(j)}$ and $\boldsymbol{d}^{(N+j)}$ for $j=1,2, \ldots, N$; in this case we also aim to take advantage of the presence of $\mathcal{V}_{\alpha}$ and target the structure of (3.19).

\subsection{A transformation}

First, we show that $\mathcal{A}^{-1} \boldsymbol{f}$ can be expressed in terms of solutions of (5.2). To achieve this we choose the generating functions

$$
\boldsymbol{g}^{(N+j)}=\mathrm{i}(\beta-\alpha) \boldsymbol{f}_{\beta}^{(j)}, \quad h^{(j)}=\mathrm{i}(\gamma-\alpha) \boldsymbol{f}_{\gamma}^{(j)} \quad(j=1,2, \ldots, N),
$$

where $\beta$ and $\gamma$ are real parameters, different from $\alpha$. Since

$$
\mathcal{V}_{\alpha} \boldsymbol{f}_{\beta}^{(j)}=\mathrm{i}(\beta-\alpha)^{-1}\left(\boldsymbol{f}_{\beta}^{(j)}-\boldsymbol{f}_{\alpha}^{(j)}\right)
$$

is easily verified (and is an example of a relationship between the set $\mathcal{V}_{\alpha} \boldsymbol{g}^{(j)}$ and the set $\left.\boldsymbol{g}^{(j)}\right)$, the transformed vectors given by (4.5) are

$$
\hat{\boldsymbol{c}}^{(N+j)}=\phi_{\alpha}^{(j)}-\mathcal{W}_{\beta, \alpha} \phi_{\beta}^{(j)}, \quad \hat{\boldsymbol{d}}^{(j)}=\boldsymbol{\psi}_{\alpha}^{(j)}-\mathcal{W}_{\gamma, \alpha} \boldsymbol{\psi}_{\gamma}^{(j)} \quad(j=1,2, \ldots, N),
$$

where

$$
\mathcal{W}_{\beta, \alpha}=\mathcal{I}-\mathrm{i}(\beta-\alpha) \mathcal{V}_{\alpha}^{*}
$$

Referring to (4.7) and writing $G$ and $H$ in terms of the $N \times N$ block matrices $G_{m n}$ and $H_{m n}$, for $m, n=1,2$, we have

$$
G=\left(\begin{array}{ll}
I & G_{12} \\
0 & G_{22}
\end{array}\right), \quad H=\left(\begin{array}{ll}
H_{11} & 0 \\
H_{21} & I
\end{array}\right),
$$

which ensure that

$$
\hat{\boldsymbol{c}}^{(j)}=\boldsymbol{c}^{(j)}=\boldsymbol{\phi}_{\alpha}^{(j)} \quad \text { and } \quad \hat{\boldsymbol{d}}^{(N+j)}=\boldsymbol{d}^{(N+j)}=\boldsymbol{\psi}_{\alpha}^{(j)}
$$

for $j=1,2, \ldots, N$, as required. Thus the transformation (4.7) can be written in the present case as

$$
\left.\begin{array}{rl}
\left(\boldsymbol{c}^{(1)}, \ldots, \boldsymbol{c}^{(2 N)}\right) & =\left(\Phi_{\alpha}, \Phi_{\alpha}-\mathcal{W}_{\beta, \alpha} \Phi_{\beta}\right) G^{-1}, \\
\left(\boldsymbol{d}^{(1)}, \ldots, \boldsymbol{d}^{(2 N)}\right) & =\left(\Psi_{\alpha}-\mathcal{W}_{\gamma, \alpha} \Phi_{\gamma}, \Psi_{\alpha}\right) H^{-1},
\end{array}\right\}
$$

where

$$
\Phi_{\alpha}=\left(\phi_{\alpha}^{(1)}, \phi_{\alpha}^{(2)}, \ldots, \phi_{\alpha}^{(N)}\right), \quad \Psi_{\alpha}=\left(\boldsymbol{\psi}_{\alpha}^{(1)}, \boldsymbol{\psi}_{\alpha}^{(2)}, \ldots, \boldsymbol{\psi}_{\alpha}^{(N)}\right),
$$

are $N \times N$ matrices. 
The matrix $\hat{G}$ occurring in (4.9) is given here by

$$
\hat{G}=H^{*} G=\left(\begin{array}{cc}
\hat{G}_{11} & \hat{G}_{12} \\
0 & \hat{G}_{22}
\end{array}\right),
$$

where

$$
\hat{G}_{11}=H_{11}^{*}, \quad \hat{G}_{12}=H_{11}^{*} G_{12}+H_{21}^{*} G_{22} \quad \text { and } \quad \hat{G}_{22}=G_{22} .
$$

The $i, j$ components of the relevant matrices are

$$
\left(\hat{G}_{11}\right)_{i j}=\left(\boldsymbol{c}^{(j)}, \boldsymbol{h}^{(i)}\right), \quad\left(\hat{G}_{12}\right)_{i j}=\left(\boldsymbol{g}^{(N+j)}, \hat{\boldsymbol{d}}^{(i)}\right), \quad\left(\hat{G}_{22}\right)_{i j}=\left(\boldsymbol{g}^{(N+j)}, \boldsymbol{d}^{(N+i)}\right) .
$$

Substituting for the vectors in the inner products and using (5.3) to simplify the $\hat{G}_{12}$ term, we can conveniently express all of these components in terms of the quantity

$$
g_{i j}(\alpha, \beta)=\mathrm{i}(\beta-\alpha)\left(\boldsymbol{f}_{\beta}^{(j)}, \boldsymbol{\psi}_{\alpha}^{(i)}\right)=\mathrm{i}(\beta-\alpha)\left(\boldsymbol{\phi}_{\beta}^{(j)}, \boldsymbol{f}_{\alpha}^{(i)}\right),
$$

where the reciprocal relations

$$
\left(\boldsymbol{f}_{\alpha}^{(j)}, \boldsymbol{\psi}_{\beta}^{(i)}\right)=\left(\boldsymbol{\phi}_{\alpha}^{(j)}, \boldsymbol{f}_{\beta}^{(i)}\right) \quad(i, j=1,2, \ldots, N)
$$

for (5.2) have been used. Thus we find that

$\left(\hat{G}_{11}\right)_{i j}=g_{i j}(\gamma, \alpha), \quad\left(\hat{G}_{12}\right)_{i j}=g_{i j}(\alpha, \beta)-g_{i j}(\gamma, \beta)+g_{i j}(\gamma, \alpha), \quad\left(\hat{G}_{22}\right)_{i j}=g_{i j}(\alpha, \beta)$.

It follows that $\hat{G}=\hat{G}(\alpha, \beta, \gamma)$ can be evaluated from a knowledge of $\phi_{\alpha}^{(j)}$ and $\phi_{\beta}^{(j)}$ for $j=1,2, \ldots, N$.

Referring to (5.4), we deduce that $\mathcal{F}^{\prime}$ is determined in this case by the $4 N$ vectors $\phi_{\alpha}^{(j)}, \boldsymbol{\psi}_{\alpha}^{(j)}, \boldsymbol{\phi}_{\beta}^{(j)}$ and $\boldsymbol{\psi}_{\gamma}^{(j)}$ for $j=1,2, \ldots, N$, for any chosen parameter values of $\alpha, \beta$ and $\gamma$ with $\alpha \neq \beta$; the choice $\beta=\gamma$ is not excluded. This transformation therefore does not increase the number of vectors required to form the inverse of $\mathcal{A}$, partly because $2 N$ of the original vectors remain intact and partly because of the choice of $\boldsymbol{g}^{(j)}$ and $\boldsymbol{h}^{(j)}$. From a practical viewpoint, there is the extra saving that only the $2 N$ equations $(5.2)$ need to be solved, albeit for two different parameter values. The equivalent economy, which also applies in the general case when the corresponding transformation is used, is the reason for incorporating the free parameter $\alpha$ into the overall structure.

A particular application of this transformation is the evaluation of $\boldsymbol{\phi}_{\delta}^{(j)}=\mathcal{A}^{-1} \boldsymbol{f}_{\delta}^{(j)}$ for any real value $\delta$ not used in the determination of $\mathcal{A}^{-1} \boldsymbol{f}$. However, a relationship between the solutions of (5.2) for different parameter values can be found directly, without recourse to the inverse operator. This is achieved by noting that the first equation in (5.4) implies that

$$
\left(\boldsymbol{c}^{(N+1)}, \ldots, \boldsymbol{c}^{(2 N)}\right) G_{22}(\alpha, \beta)=\left(\Phi_{\alpha}-\mathcal{W}_{\beta, \alpha} \Phi_{\beta}\right)-\Phi_{\alpha} G_{12}(\alpha, \beta),
$$

when the dependence of the matrices $G_{m n}$ on the parameters is made explicit. Now $G_{12}(\alpha, \beta)$ and $\left(\boldsymbol{c}^{(N+1)}, \ldots, \boldsymbol{c}^{(2 N)}\right)$ are not expressed in terms of the solutions of $(5.2)$ and must therefore be eliminated from the proceedings. Using a second parameter value, which we again denote by $\gamma$, we first remove the matrix $\left(\boldsymbol{c}^{(N+1)}, \ldots, \boldsymbol{c}^{(2 N)}\right)$ to give

$$
\Phi_{\alpha} E(\alpha, \beta, \gamma)=\mathcal{W}_{\gamma, \alpha} \Phi_{\gamma} G_{22}^{-1}(\alpha, \gamma)-\mathcal{W}_{\beta, \alpha} \Phi_{\beta} G_{22}^{-1}(\alpha, \beta),
$$


where

$$
E(\alpha, \beta, \gamma)=G_{22}^{-1}(\alpha, \gamma)-G_{22}^{-1}(\alpha, \beta)-G_{12}(\alpha, \gamma) G_{22}^{-1}(\alpha, \gamma)+G_{12}(\alpha, \beta) G_{22}^{-1}(\alpha, \beta) .
$$

Multiplying both sides of $(5.5)$ by the matrix $\left(\boldsymbol{f}_{-\omega}^{(1)}, \ldots, \boldsymbol{f}_{-\omega}^{(N)}\right)$, for some $\omega$, and integrating results in the formula

$$
G_{22}(\omega, \alpha) E(\alpha, \beta, \gamma)=G_{22}(\omega, \gamma) G_{22}^{-1}(\alpha, \gamma)-G_{22}(\omega, \beta) G_{22}^{-1}(\alpha, \beta),
$$

in which (5.3) has been used to show that

$$
\left(\mathcal{W}_{\beta, \alpha} \phi_{\beta}^{(j)}, \boldsymbol{f}_{\omega}^{(i)}\right)=(\alpha-\omega)^{-1}\left\{(\beta-\omega)\left(\phi_{\beta}^{(j)}, \boldsymbol{f}_{\omega}^{(i)}\right)-(\beta-\alpha)\left(\phi_{\beta}^{(j)}, \boldsymbol{f}_{\alpha}^{(i)}\right)\right\} .
$$

Setting $\omega=\gamma$ and $\omega=\beta$ in (5.6) in turn and using the property $G_{22}(\beta, \beta)=0$ we obtain two alternative, equivalent versions of $E$ that do not contain $G_{12}$, namely,

$$
\begin{aligned}
E(\alpha, \beta, \gamma) & =-G_{22}^{-1}(\gamma, \alpha) G_{22}(\gamma, \beta) G_{22}^{-1}(\alpha, \beta) \\
& =G_{22}^{-1}(\beta, \alpha) G_{22}(\beta, \gamma) G_{22}^{-1}(\alpha, \gamma) .
\end{aligned}
$$

When these are used in (5.5) they give

$$
\Phi_{\alpha}=\mathcal{W}_{\gamma, \alpha} \Phi_{\gamma} G_{22}^{-1}(\beta, \gamma) G_{22}(\beta, \alpha)+\mathcal{W}_{\beta, \alpha} \Phi_{\beta} G_{22}^{-1}(\gamma, \beta) G_{22}(\gamma, \alpha)
$$

and (5.6) becomes

$$
G_{22}(\omega, \alpha)=G_{22}(\omega, \gamma) G_{22}^{-1}(\beta, \gamma) G_{22}(\beta, \alpha)+G_{22}(\omega, \beta) G_{22}^{-1}(\gamma, \beta) G_{22}(\gamma, \alpha) .
$$

Equations (5.7) and (5.8) are examples of embedding formulae for the system if their right-hand sides can be constructed independently of $\phi_{\alpha}^{(1)}, \ldots, \phi_{\alpha}^{(N)}$, and this is indeed possible. Thus (5.7) expresses $\phi_{\alpha}^{(j)}$ for each $j$ and any $\alpha$ in terms of $\phi_{\beta}^{(j)}, \phi_{\gamma}^{(j)}, \boldsymbol{\psi}_{\beta}^{(j)}$ and $\boldsymbol{\psi}_{\gamma}^{(j)}$ for $j=1,2, \ldots, N$, provided that $\beta \neq \gamma$. The same solutions are required in (5.7) to determine $G_{22}(\omega, \alpha)$, with $i, j$ component $\mathrm{i}(\alpha-\omega)\left(\phi_{\alpha}^{(j)}, \boldsymbol{f}_{\omega}^{(i)}\right)$, for any $\alpha$ and any $\omega \neq \alpha$. Such projections of $\phi^{(j)}$ are often significant in practical examples of the system (2.1).

The situation described above is typical of embedding results, for example those obtained recently by Biggs et al. [3] and Biggs and Porter $[\mathbf{1}, \mathbf{2}]$ for specific integralequation systems. On making the necessary notational adjustments and specializing to the case considered by Biggs and Porter [2], it can be verified that (5.7) and (5.8) coincide with formulae derived in that paper. The embedding results obtained here apply to the general equation (2.5), of course, and they are special cases of the present investigation in that they avoid the need to invoke a formula for $\mathcal{A}^{-1}$.

\subsection{A different transformation}

We now examine the alternative transformation of the vectors $\boldsymbol{c}^{(j)}$ and $\boldsymbol{d}^{(j)}$ indicated earlier. Our objective here is to retain the vectors $\boldsymbol{S}^{(j)}$ and $\boldsymbol{T}^{(j)}$ and to replace $\boldsymbol{c}^{(j)}=\boldsymbol{\phi}_{\alpha}^{(j)}$ and $\boldsymbol{d}^{(N+j)}=\boldsymbol{\psi}_{\alpha}^{(j)}$. However, rather than use $\boldsymbol{c}^{(N+j)}$ and $\boldsymbol{d}^{(j)}$ as they stand, we also seek 
a representation of $\mathcal{A}^{-1}$ in which the vectors satisfy (3.19). This further objective can also be achieved by adapting the basic generating formulae (4.5).

Setting $\boldsymbol{g}^{(j)}=\boldsymbol{S}^{(j)}$ and $\boldsymbol{h}^{(j)}=\boldsymbol{T}^{(j)}$ for $j=1,2, \ldots, N$, those formulae give

$$
\hat{\boldsymbol{c}}^{(j)}=-\lambda_{j} w_{j} \tilde{\boldsymbol{c}}^{(j)}-\boldsymbol{c}^{(N+j)}, \quad \hat{\boldsymbol{d}}^{(j)}=\tilde{\boldsymbol{d}}^{(j)}+w_{j} \bar{\lambda}_{j} \boldsymbol{d}^{(N+j)}-\boldsymbol{d}^{(j)},
$$

using the notation of (5.1), where the new vectors

$$
\tilde{\boldsymbol{c}}^{(j)}=-\left(\lambda_{j} w_{j}\right)^{-1} \mathcal{V}_{\alpha}^{*} \mathcal{A}^{-1} \boldsymbol{S}^{(j)}, \quad \tilde{\boldsymbol{d}}^{(j)}=\mathcal{V}_{\alpha}^{*} \mathcal{A}^{*-1} \boldsymbol{T}^{(j)}
$$

have the desired structure. The form of $\tilde{\boldsymbol{c}}^{(j)}$ has been chosen for later algebraic convenience.

Natural replacements for $\boldsymbol{c}^{(j)}$ and $\boldsymbol{d}^{(N+j)}$ are $\boldsymbol{P}^{(j)}$ and $\boldsymbol{Q}^{(j)}(j=1,2, \ldots, N)$, the counterparts of $\boldsymbol{S}^{(j)}$ and $\boldsymbol{T}^{(j)}$ in (2.12). Indeed, if $\mathcal{A}^{-1}$ is determined by taking (2.12) as the starting point, rather than $(2.11)$, then $\boldsymbol{P}^{(j)}$ and $\boldsymbol{Q}^{(j)}$ will clearly play a prominent part in the calculation. Here we are exploiting the interplay between (2.11) and (2.12), which are related through (2.15) and (2.16). We therefore complete the transformation by choosing $\boldsymbol{g}^{(N+j)}=\mathrm{e}^{-\mathrm{i} \alpha w_{j}} \boldsymbol{P}^{(j)}$ and $\boldsymbol{h}^{(N+j)}=\mathrm{e}^{-\mathrm{i} \alpha w_{j}} \boldsymbol{Q}^{(j)}$ for $j=1,2, \ldots, N$, noting that

$$
\begin{aligned}
& \mathcal{A}^{-1}\left\{\mathrm{e}^{-\mathrm{i} \alpha w_{j}} \mathcal{V}_{\alpha} \boldsymbol{P}^{(j)}\right\}=w_{j} \boldsymbol{f}_{\alpha}^{(j)}-w_{j} \lambda_{j} \boldsymbol{c}^{(j)}-\boldsymbol{c}^{(N+j)}+w_{j} \sum_{i=1}^{N}\left(\boldsymbol{f}_{\alpha}^{(j)}, \boldsymbol{h}^{(i)}\right) \boldsymbol{c}^{(i)}, \\
& \mathcal{A}^{*-1}\left\{\mathrm{e}^{-\mathrm{i} \alpha w_{j}} \mathcal{V}_{\alpha} \boldsymbol{Q}^{(j)}\right\}=w_{j} \boldsymbol{f}_{\alpha}^{(j)}-\boldsymbol{d}^{(j)}+w_{j} \sum_{i=1}^{N}\left(\boldsymbol{f}_{\alpha}^{(j)}, \boldsymbol{g}^{(i)}\right) \boldsymbol{d}^{(N+i)}
\end{aligned}
$$

follow from (2.16) and (5.1).

These equations suggest that we take

$$
\left.\begin{array}{l}
\tilde{\boldsymbol{c}}^{(N+j)}=\left(\lambda_{j} w_{j}\right)^{-1}\left\{\mathrm{e}^{-\mathrm{i} \alpha w_{j}} \mathcal{V}_{\alpha}^{*} \mathcal{A}^{-1} \boldsymbol{P}^{(j)}+w_{j} \boldsymbol{f}_{\alpha}^{(j)}\right\} \\
\tilde{\boldsymbol{d}}^{(N+j)}=\mathrm{e}^{-\mathrm{i} \alpha w_{j}} \mathcal{V}_{\alpha}^{*} \mathcal{A}^{*-1} \boldsymbol{Q}^{(j)}+w_{j} \boldsymbol{f}_{\alpha}^{(j)}
\end{array}\right\} \quad(j=1,2, \ldots, N)
$$

to parallel (5.10) and complete the transformation and, using (4.5), we find that

$$
\left.\begin{array}{l}
\hat{\boldsymbol{c}}^{(N+j)}=\lambda_{j} w_{j}\left(\tilde{\boldsymbol{c}}^{(N+j)}-\boldsymbol{c}^{(j)}\right)-\boldsymbol{c}^{(N+j)}+w_{j} \sum_{i=1}^{N}\left(\boldsymbol{f}_{\alpha}^{(j)}, \boldsymbol{h}^{(i)}\right) \boldsymbol{c}^{(i)}, \\
\hat{\boldsymbol{d}}^{(N+j)}=\tilde{\boldsymbol{d}}^{(N+j)}-\boldsymbol{d}^{(j)}+w_{j} \sum_{i=1}^{N}\left(\boldsymbol{f}_{\alpha}^{(j)}, \boldsymbol{g}^{(i)}\right) \boldsymbol{d}^{(N+i)},
\end{array}\right\}
$$

for $j=1,2, \ldots, N$.

The transformation we now envisage is from $\boldsymbol{c}^{(j)}$ and $\boldsymbol{d}^{(j)}$ into the sets of vectors $\tilde{\boldsymbol{c}}^{(j)}$ and $\tilde{\boldsymbol{d}}^{(j)}$. It is implemented through Equations (5.9) and (5.12), which may be written in matrix form as

$$
\left.\begin{array}{rl}
\left(\hat{\boldsymbol{c}}^{(1)}, \ldots, \hat{\boldsymbol{c}}^{(2 N)}\right) & =\left(\tilde{\boldsymbol{c}}^{(1)}, \ldots, \tilde{\boldsymbol{c}}^{(2 N)}\right) J-\left(\boldsymbol{c}^{(1)}, \ldots, \boldsymbol{c}^{(2 N)}\right) G_{1}, \\
\left(\hat{\boldsymbol{d}}^{(1)}, \ldots, \hat{\boldsymbol{d}}^{(2 N)}\right) & =\left(\tilde{\boldsymbol{d}}^{(1)}, \ldots, \tilde{\boldsymbol{d}}^{(2 N)}\right)-\left(\boldsymbol{d}^{(1)}, \ldots, \boldsymbol{d}^{(2 N)}\right) H_{1},
\end{array}\right\}
$$


where

$$
J=\left(\begin{array}{cc}
-W \Lambda & 0 \\
0 & W \Lambda
\end{array}\right), \quad G_{1}=\left(\begin{array}{cc}
0 & W \Lambda-F_{1} W \\
I & I
\end{array}\right), \quad H_{1}=\left(\begin{array}{cc}
I & I \\
-W \bar{\Lambda} & -F_{2} W
\end{array}\right) .
$$

Here $F_{1}$ and $F_{2}$ are the $N \times N$ matrices with $i, j$ components $\left(\boldsymbol{f}_{\alpha}^{(j)}, \boldsymbol{h}^{(i)}\right)$ and $\left(\boldsymbol{f}_{\alpha}^{(j)}, \boldsymbol{g}^{(i)}\right)$, respectively. The matrices $W=\operatorname{diag}\left(w_{1}, w_{2}, \ldots, w_{N}\right)$ and $\Lambda=\operatorname{diag}\left(\lambda_{1}, \lambda_{2}, \ldots, \lambda_{N}\right)$ have been used earlier.

Now (5.13) can be expressed as

$$
\left.\begin{array}{rl}
\left(\boldsymbol{c}^{(1)}, \ldots, \boldsymbol{c}^{(2 N)}\right)\left(G+G_{1}\right) J^{-1} & =\left(\tilde{\boldsymbol{c}}^{(1)}, \ldots, \tilde{\boldsymbol{c}}^{(2 N)}\right), \\
\left(\boldsymbol{d}^{(1)}, \ldots, \boldsymbol{d}^{(2 N)}\right)\left(H+H_{1}\right) & =\left(\tilde{\boldsymbol{d}}^{(1)}, \ldots, \tilde{\boldsymbol{d}}^{(2 N)}\right)
\end{array}\right\}
$$

by invoking (4.7), and therefore the counterpart of (4.9) is

$$
\mathcal{F}^{\prime} \boldsymbol{f}=\left(\tilde{\boldsymbol{c}}^{(1)}, \ldots, \tilde{\boldsymbol{c}}^{(2 N)}\right) G^{-1}\left(\left(\boldsymbol{f}, \tilde{\boldsymbol{d}}^{(1)}\right), \ldots,\left(\boldsymbol{f}, \tilde{\boldsymbol{d}}^{(2 N)}\right)\right)^{\mathrm{T}},
$$

in which $G=\left(H+H_{1}\right)^{*}\left(G+G_{1}\right) J^{-1}$ has to be evaluated to complete the transformation.

We readily deduce from (5.13) that

$$
\hat{H}=J^{*} \tilde{H}-G_{1}^{*} H, \quad \hat{G}=\tilde{G}-H_{1}^{*} G,
$$

where $G, H, \hat{G}$ and $\hat{H}$ are defined in $\S$ 4.1. The new matrices $\tilde{G}$ and $\tilde{H}$ are obvious extensions of the earlier notation, having $i, j$ components $\left(\boldsymbol{g}^{(j)}, \tilde{\boldsymbol{d}}^{(i)}\right)$ and $\left(\boldsymbol{h}^{(j)}, \tilde{\boldsymbol{c}}^{(i)}\right)$, respectively. However, a relationship between $\tilde{G}$ and $\tilde{H}$ does not follow from the transformation structure of the previous section and has to be evaluated at this stage. Now

$$
\left(\boldsymbol{g}^{(j)}, \tilde{\boldsymbol{d}}^{(i)}\right)=\left(\boldsymbol{S}^{(j)}, \mathcal{V}_{\alpha}^{*} \mathcal{A}^{*-1} \boldsymbol{T}^{(i)}\right)=\left(\mathcal{A}^{-1} \mathcal{V}_{\alpha} \boldsymbol{S}^{(j)}, \boldsymbol{T}^{(i)}\right)=\left(\hat{\boldsymbol{c}}^{(j)}+w_{j} \lambda_{j} \tilde{\boldsymbol{c}}^{(j)}, \boldsymbol{h}^{(i)}\right),
$$

for $i, j=1,2, \ldots, N$. When this and similar calculations encompassing $i, j=1,2, \ldots, 2 N$ are used with (5.13) and (2.14), we find that

$$
\tilde{G}+\tilde{H}^{*} J=\hat{H}^{*}+J-H_{1}^{*} G_{1} .
$$

Eliminating $\tilde{G}$ and $\tilde{H}$ between this equation and (5.16) and recalling that $\hat{H}^{*}=\hat{G}=$ $H^{*} G$, it follows that

$$
\left\{H+H_{1}\right\}^{*}\left\{G+G_{1}\right\}=J
$$

and therefore $G=I$. Thus, (4.1) and (5.15) imply that

$$
\mathcal{V}_{\alpha}^{*} \mathcal{A}^{-1} \boldsymbol{f}+\mathcal{A}^{-1} \mathcal{V}_{\alpha} \boldsymbol{f}=\mathcal{F}^{\prime} \boldsymbol{f}=\sum_{j=1}^{2 N}\left(\boldsymbol{f}, \tilde{\boldsymbol{d}}^{(j)}\right) \tilde{\boldsymbol{c}}^{(j)},
$$

showing that the form of (4.1) is invariant under the particular transformation devised here. The factors $\pm\left(\lambda_{j} w_{j}\right)^{-1}$ introduced into $\tilde{\boldsymbol{c}}^{(j)}$ anticipated this outcome; without them $G$ is diagonal and a later scaling achieves (5.17). 
It follows from (5.10) and (5.11) that the vectors defining this transformed version of $\mathcal{F}^{\prime}$ are given in terms of

$$
\boldsymbol{s}^{(j)}=\mathcal{A}^{-1} \boldsymbol{S}^{(j)}, \quad \boldsymbol{p}^{(j)}=\mathcal{A}^{-1} \boldsymbol{P}^{(j)}, \quad \boldsymbol{t}^{(j)}=\mathcal{A}^{*-1} \boldsymbol{T}^{(j)}, \quad \boldsymbol{q}^{(j)}=\mathcal{A}^{*-1} \boldsymbol{Q}^{(j)}
$$

as

$$
\left.\begin{array}{ll}
\tilde{\boldsymbol{c}}^{(j)}=-\left(\lambda_{j} w_{j}\right)^{-1} \mathcal{V}_{\alpha}^{*} \boldsymbol{s}^{(j)}, & \tilde{\boldsymbol{c}}^{(N+j)}=\left(\lambda_{j} w_{j}\right)^{-1}\left\{\mathrm{e}^{-\mathrm{i} \alpha w_{j}} \mathcal{V}_{\alpha}^{*} \boldsymbol{p}^{(j)}+w_{j} \boldsymbol{f}_{\alpha}^{(j)}\right\}, \\
\tilde{\boldsymbol{d}}^{(j)}=\mathcal{V}_{\alpha}^{*} \boldsymbol{t}^{(j)}, & \tilde{\boldsymbol{d}}^{(N+j)}=\mathrm{e}^{-\mathrm{i} \alpha w_{j}} \mathcal{V}_{\alpha}^{*} \boldsymbol{q}^{(j)}+w_{j} \boldsymbol{f}_{\alpha}^{(j)},
\end{array}\right\}
$$

for $j=1,2, \ldots, N$. Once again there is no increase in the overall number of vectors needed to construct $\mathcal{A}^{-1} \boldsymbol{f}$. The particular significance of the set of vectors (5.19) is that it is formed through (5.18) from the 'boundary values' of the kernels occurring in (2.1), as reference to (2.9) and (2.13) shows.

By construction, $\tilde{\boldsymbol{c}}^{(j)}$ and $\tilde{\boldsymbol{d}}^{(j)}$ satisfy (3.19) and therefore an explicit version of $\mathcal{A}^{-1} \boldsymbol{f}$ having the form of (3.20) is guaranteed. Substituting (5.19) into that equation confirms that (4.3) applies for components of $\mathcal{A}^{-1} \boldsymbol{f}$. Term-by-term differentiation of the corresponding version of (3.22) gives the components of the resolvent kernel as

$$
\begin{array}{r}
r_{m n}^{\prime}(x, t)=z_{m n}^{\prime}(x, t)-\sum_{j=1}^{N} \frac{w_{m} w_{n}}{w_{j} \lambda_{j}} \int_{0}^{\ell(1-x, 1-t)}\left\{s _ { m } ^ { ( j ) } \left(x+w_{n} \sigma \overline{t_{n}^{(j)}\left(t+w_{m} \sigma\right)}\right.\right. \\
\left.-p_{m}^{(j)}\left(x+w_{n} \sigma\right) \overline{q_{n}^{(j)}\left(t+w_{m} \sigma\right)}\right\} \mathrm{d} \sigma,
\end{array}
$$

where

$$
z_{m n}^{\prime}(x, t)= \begin{cases}\lambda_{m}^{-1} \overline{q_{n}^{(m)}\left(t+w_{m}(1-x) / w_{n}\right)} & \left(w_{m}(1-x)<w_{n}(1-t)\right), \\ \lambda_{n}^{-1} p_{m}^{(n)}\left(x+w_{n}(1-t) / w_{m}\right) & \left(w_{n}(1-t)<w_{m}(1-x)\right),\end{cases}
$$

for almost all $x, t \in[0,1] \times[0,1]$.

We now know that the transformed vectors constructed in $\S 5.1$ are continuous in $[0,1]$ and have derivatives in $L_{2}(0,1)$ since they are expressible in terms of $\tilde{\boldsymbol{c}}^{(j)}$ and $\tilde{\boldsymbol{d}}^{(j)}$ through two successive transformations. More generally, the original vectors $\boldsymbol{c}^{(j)}$ and $\boldsymbol{d}^{(j)}$ and all other transformations of them have these properties.

The identity (2.15) may be used to provide a different type of transformation of the vectors needed to construct $\mathcal{A}^{-1}$, as we can illustrate in the present context. It can be rearranged as

$$
\begin{aligned}
\left(\mathcal{V}_{\alpha} \mathcal{A}^{-1}+\mathcal{A}^{-1} \mathcal{V}_{\alpha}^{*}\right) \boldsymbol{f}+\left(\mathcal{V}_{\alpha}^{*} \mathcal{A}^{-1}+\mathcal{A}^{-1} \mathcal{V}_{\alpha}\right) \boldsymbol{f} & \\
& =\sum_{j=1}^{N} w_{j}\left\{\left(\boldsymbol{f}, \boldsymbol{f}_{\alpha}^{(j)}\right) \mathcal{A}^{-1} \boldsymbol{f}_{\alpha}^{(j)}+\left(\boldsymbol{f}, \mathcal{A}^{*-1} \boldsymbol{f}_{\alpha}^{(j)}\right) \boldsymbol{f}_{\alpha}^{(j)}\right\},
\end{aligned}
$$

showing that a representation of $\left(\mathcal{V}_{\alpha} \mathcal{A}^{-1}+\mathcal{A}^{-1} \mathcal{V}_{\alpha}^{*}\right) \boldsymbol{f}$ can be deduced from each representation of $\left(\mathcal{V}_{\alpha}^{*} \mathcal{A}^{-1}+\mathcal{A}^{-1} \mathcal{V}_{\alpha}\right) \boldsymbol{f}$. In particular, we can determine the representation

$$
\left(\mathcal{V}_{\alpha} \mathcal{A}^{-1}+\mathcal{A}^{-1} \mathcal{V}_{\alpha}^{*}\right) \boldsymbol{f} \equiv \mathcal{F} \boldsymbol{f}=\sum_{j=1}^{2 N}\left(\boldsymbol{f}, \tilde{\boldsymbol{b}}^{(j)}\right) \tilde{\boldsymbol{a}}^{(j)},
$$


which is implied by (5.17). To evaluate $\tilde{\boldsymbol{a}}^{(j)}$ and $\tilde{\boldsymbol{b}}^{(j)}$, we note that

$$
\mathcal{A}^{-1} \boldsymbol{f}_{\alpha}^{(j)}=\phi_{\alpha}^{(j)}=\boldsymbol{c}^{(j)} \quad \text { and } \quad \mathcal{A}^{*-1} \boldsymbol{f}_{\alpha}^{(j)}=\boldsymbol{\psi}_{\alpha}^{(j)}=\boldsymbol{d}^{(N+j)}
$$

in the notation of (5.1). Thus (5.17) and (5.21) give

$$
\mathcal{F} \boldsymbol{f}=\sum_{j=1}^{N} w_{j}\left\{\left(\boldsymbol{f}, \boldsymbol{f}_{\alpha}^{(j)}\right) \boldsymbol{c}^{(j)}+\left(\boldsymbol{f}, \boldsymbol{d}^{(N+j)}\right) \boldsymbol{f}_{\alpha}^{(j)}\right\}-\sum_{j=1}^{2 N}\left(\boldsymbol{f}, \tilde{\boldsymbol{d}}^{(j)}\right) \tilde{\boldsymbol{c}}^{(j)} .
$$

We can replace $\boldsymbol{c}^{(1)}, \ldots, \boldsymbol{c}^{(N)}$ by $\tilde{\boldsymbol{c}}^{(1)}, \ldots, \tilde{\boldsymbol{c}}^{(2 N)}$ and $\boldsymbol{d}^{(N+1)}, \ldots, \boldsymbol{d}^{(2 N)}$ by $\tilde{\boldsymbol{d}}^{(1)}, \ldots, \tilde{\boldsymbol{d}}^{(2 N)}$ and using the established properties of the matrices occurring in (5.14), we find after some manipulation that

$$
\left.\begin{array}{ll}
\tilde{\boldsymbol{a}}^{(j)}=\left(\lambda_{j} w_{j}\right)^{-1}\left\{\mathcal{V}_{\alpha} \boldsymbol{s}^{(j)}+w_{j} \boldsymbol{f}_{\alpha}^{(j)}\right\}, & \tilde{\boldsymbol{a}}^{(N+j)}=-\left(\lambda_{j} w_{j}\right)^{-1} \mathrm{e}^{-\mathrm{i} \alpha w_{j}} \mathcal{V}_{\alpha} \boldsymbol{p}^{(j)}, \\
\tilde{\boldsymbol{b}}^{(j)}=\mathcal{V}_{\alpha} \boldsymbol{t}^{(j)}+w_{j} \boldsymbol{f}_{\alpha}^{(j)}, & \tilde{\boldsymbol{b}}^{(N+j)}=\mathrm{e}^{-\mathrm{i} \alpha w_{j}} \mathcal{V}_{\alpha} \boldsymbol{q}^{(j)} .
\end{array}\right\}
$$

The representation of $\mathcal{F}$ given by (5.22) and (5.23) can alternatively be deduced by taking (2.12) as the starting point for the investigation of the integral-equation system, rather than (2.11), and implementing the procedure which led to (5.20). The significance of (5.22) is that it has the form (2.17) and therefore leads to a formula for the components of the resolvent different from (5.20). Inevitably, the vectors (5.23) are examples of the structure (3.11). Thus (3.13) applies, giving

$$
\left(\mathcal{A}^{-1} \boldsymbol{f}\right)_{m}(x)=\lambda_{m}^{-1} f_{m}(x)+\sum_{n=1}^{N} \int_{0}^{1} r_{m n}(x, t) f_{n}(t) \mathrm{d} t
$$

for $m=1,2, \ldots, N$, where

$$
\begin{array}{r}
r_{m n}(x, t)=z_{m n}(x, t)-\sum_{j=1}^{N} \frac{w_{m} w_{n}}{w_{j} \lambda_{j}} \int_{0}^{\ell(x, t)}\left\{p_{m}^{(j)}\left(x-w_{n} \sigma\right) \overline{q_{n}^{(j)}\left(t-w_{m} \sigma\right)}\right. \\
\left.-s_{m}^{(j)}\left(x-w_{n} \sigma\right) \overline{t_{n}^{(j)}\left(t-w_{m} \sigma\right)}\right\} \mathrm{d} \sigma
\end{array}
$$

where

$$
z_{m n}(x, t)= \begin{cases}\lambda_{m}^{-1} \overline{t_{n}^{(m)}\left(t-w_{m} x / w_{n}\right)} & \left(w_{m} x<w_{n} t\right), \\ \lambda_{n}^{-1} s_{m}^{(n)}\left(x-w_{n} t / w_{m}\right) & \left(w_{n} t<w_{m} x\right),\end{cases}
$$

for almost all $x, t \in[0,1] \times[0,1]$.

Although we have preserved the earlier notation for clarity, $r_{m n}^{\prime}(x, t)$ and $r_{m n}(x, t)$ are, of course, different representations of the same functions.

\subsection{Special cases}

If $w_{n}=1$ for $n=1,2, \ldots, N,(2.1)$ reduces to a system of equations with pure difference kernels. This permits a more concise notation to be used than in the general case, in which the vectors $\boldsymbol{s}^{(j)}$ defined in (5.18) are gathered into the $N \times N$ matrix

$$
S=\left(\boldsymbol{s}^{(1)}, \ldots, \boldsymbol{s}^{(N)}\right),
$$

with corresponding definitions of $P, T$ and $Q$. 
The resolvent matrix $R^{\prime}(x, t)$ with $m, n$ component given by (5.20) can therefore be written as

$R^{\prime}(x, t)=Z^{\prime}(x, t)-\int_{0}^{\min (1-x, 1-t)}\left\{S(x+\sigma) \Lambda^{-1} T^{*}(t+\sigma)-P(x+\sigma) \Lambda^{-1} Q^{*}(t+\sigma)\right\} \mathrm{d} \sigma$,

where

$$
Z^{\prime}(x, t)= \begin{cases}\Lambda^{-1} Q^{*}(1+t-x) & (t \leqslant x), \\ P(1+x-t) \Lambda^{-1} & (x<t) .\end{cases}
$$

(The asterisk here denotes the Hermitian transpose.)

The alternative form (5.24) of the resolvent similarly condenses to

$$
R(x, t)=Z(x, t)-\int_{0}^{\min (x, t)}\left\{P(x-\sigma) \Lambda^{-1} Q^{*}(t-\sigma)-S(x-\sigma) \Lambda^{-1} T^{*}(t-\sigma)\right\} \mathrm{d} \sigma,
$$

with

$$
Z(x, t)= \begin{cases}\Lambda^{-1} T^{*}(t-x) & (x<t), \\ S(x-t) \Lambda^{-1} & (t<x) .\end{cases}
$$

If we write the matrix kernel of the integral operator $\mathcal{K}$ in $(2.2)$ in this case as $K(x-t)$, we readily find that Equations (5.18) can be replaced by the matrix forms

$$
\left.\begin{array}{rlrl}
(\mathcal{A} S)(x) & =K(x), & (\mathcal{A P})(x) & =K(x-1), \\
\left(\mathcal{A}^{*} T\right)(x) & =K^{*}(-x), & \left(\mathcal{A}^{*} Q\right)(x) & =K^{*}(1-x),
\end{array}\right\}
$$

holding for almost all $x \in[0,1]$.

If the further restriction is made that $K(x)=K(-x)$ almost everywhere in $[0,1]$, it follows from (5.26) that $P(x)=S(1-x)$ and $Q(x)=T(1-x)$, for almost all $x \in[0,1]$. Using this information and putting $\Lambda=I$, we find that the version (5.25) of the resolvent coincides with that derived by Mullikin and Victory [10].

In the simplest case $N=1$ of a single integral equation with a difference kernel (not necessarily even), Equations (5.26) imply that $Q(x)=\overline{S(1-x)}$ and $P(x)=\overline{T(1-x)}$, almost everywhere in $[0,1]$. The resulting versions of both $R^{\prime}(x, t)$ and $R(x, t)$ were given by Porter $[\mathbf{1 1}]$.

\section{Conclusions}

We have extended the class of operators whose inverses are known to be finitely generated, that is, expressible in terms of finitely many functions. The class is exemplified by an operator representing a system of coupled integral equations with weighted difference kernels and such systems arise in linear boundary-value problems of a particular type.

The theory develops by considering those operators that satisfy a particular algebraic relationship, which ensures that they and their inverses are finitely generated. The actual form of the operators that are included is obtained by solving the defining relationship 
under certain hypotheses that are suggested by the particular example of the integralequation system. By deducing the solution of a related operator equation, explicit forms for the inverses of the class of operators under consideration are also determined. Moreover, the structure developed permits a transformation to be constructed which leads to different forms for the inverse operator.

We have illustrated the theory, and the transformation technique in particular, by reference to the original integral-equation system. In particular, it has been shown that the inverse operator can be expressed in this case in terms of the solutions of a set of integral-equation systems in which the free terms are formed from the boundary values (that is, the values on $x=0,1$ and $t=0,1)$ of the kernels. This generalizes a result for systems with pure difference kernels as noted in $\S 5.3$. The connection between the present approach and embedding has also been made, producing a link with Biggs and Porter $[\mathbf{2}]$.

The theory can be generalized in a number of ways. For example, it is not surprising that the finite-rank operator defined by

$$
\mathcal{K}_{F} \phi=\sum_{p=1}^{P}\left(\phi, \boldsymbol{N}^{(p)}\right) \boldsymbol{M}^{(p)}
$$

where $\boldsymbol{M}^{(p)}$ and $\boldsymbol{N}^{(p)}$ are given vectors, falls into the category that we have considered. Thus, if the operator $\mathcal{K}$ generated by weighted difference kernels and defined in $\S 2$ is replaced by $\mathcal{K}-\mathcal{K}_{F}$, then (2.11) is amended by the addition of

$$
\sum_{p=1}^{P}\left\{\left(\boldsymbol{\phi}, \boldsymbol{N}^{(p)}\right) \mathcal{V}_{\alpha} \boldsymbol{M}^{(p)}+\left(\boldsymbol{\phi}, \mathcal{V}_{\alpha} \boldsymbol{N}^{(p)}\right) \boldsymbol{M}^{(p)}\right\}
$$

and therefore $\mathcal{A}=\Lambda \mathcal{I}-\mathcal{K}+\mathcal{K}_{F}$ satisfies (2.17).

A different finite-rank perturbation arises by returning to (3.15), a formula that defines the kernels of the operators $\mathcal{A}$ satisfying (2.17), subject to the requirements that $a_{n}^{(j)}$ and $b_{n}^{(j)}$ are continuous in $[0,1]$ and have derivatives in $L_{2}(0,1)$. Differentiating the expression (3.15) for $r_{m n}$ with respect to $x$ and $t$ in turn, integrating by parts and using the notation of (3.12), we find that

$$
\left(w_{n} \frac{\partial}{\partial x}+w_{m} \frac{\partial}{\partial t}\right) r_{m n}(x, t)=w_{m} w_{n} \sum_{j=1}^{M} a_{m}^{(j)}(x) \overline{b_{n}^{(j)}(t)}
$$

holds for almost all $x, t \in[0,1] \times[0,1], a_{m}^{(j)}$ and $b_{n}^{(j)}$ being elements of $L_{2}(0,1)$. Now the homogeneous equation

$$
w_{n}\left(\frac{\partial k_{m n}}{\partial x}\right)+w_{m}\left(\frac{\partial k_{m n}}{\partial t}\right)=0
$$

has general solution $k_{m n}(x, t)=\kappa_{m n}\left(w_{m} x-w_{n} t\right)$, the weighted difference kernel used to introduce the class (2.17) of operators. We now see that the class includes operators $\mathcal{A}=\Lambda \mathcal{I}-\mathcal{K}$ in which the kernels of $\mathcal{K}$ are finite-rank departures from weighted difference 
kernels, in the sense of (6.1). That equation generalizes the starting point (1.4) taken by Kon [9], who investigated a finite-rank perturbation of a difference kernel in the scalar case. In the framework developed here, the generalization is represented by (3.14) and its consequences and we have resorted to (6.1) only for the purpose of comparison with the earlier work.

\section{References}

1. N. R. T. Biggs And D. Porter, Wave diffraction through a perforated breakwater of non-zero thickness, Q. J. Mech. Appl. Math. 54 (2001), 523-547.

2. N. R. T. Biggs And D. Porter, Wave scattering by a perforated duct, Q. J. Mech. Appl. Math. 55 (2002), 249-272.

3. N. R. T. Biggs, D. Porter And D. S. G. Stirling, Wave diffraction through a perforated breakwater, Q. J. Mech. Appl. Math. 53 (2000), 375-391.

4. S. N. Chandler-Wilde, M. Rahman and C. R. Ross, A fast two-grid and finite section method for a class of integral equations on the real line with application to an acoustic scattering problem in the half-plane, Numer. Math. 93 (2002), 1-51.

5. I. Feldman, I. Gohberg and N. Krupnik, Convolution equations on finite intervals and factorisation of matrix functions, Integ. Eqns Operat. Theory 36 (2000), 201-211.

6. I. C. Gohberg And I. A. Feldman, Convolution equations and projection methods for their solution, Translations of Mathematical Monographs, vol. 41 (American Mathematical Society, Providence, RI, 1974).

7. T. Kailath, L. LuUng and M. Morf, Generalised Krein-Levinson equations for the efficient computation of Fredholm resolvents of non-displacement kernels, in Surveys in mathematical analysis: essays dedicated to M. G. Krein (ed. I. C. Gohberg and M. Kac) (Academic, 1978).

8. I. Koltracht, B. A. Kon And L. Lerer, Inversion of structured operators, Integ. Eqns Operat. Theory 20 (1994), 410-448.

9. B. A. Kon, Inverse of Wiener-Hopf-type operators, J. Integ. Eqns Applic. 7 (1984), 93101.

10. T. W. Mullikin and D. Victory, $N$-group transport theory: a critical problem in slab geometry, J. Math. Analysis Applic. 58 (1977), 605-630.

11. D. PorTer, The solution of integral equations with difference kernels, J. Integ. Eqns Applic. 3 (1991), 429-454.

12. D. Porter And D. S. G. Stirling, Finitely-generated solutions of certain integral equations, Proc. Edinb. Math. Soc. 37 (1994), 325-345.

13. L. A. Sakhnovich, Equations with a difference kernel on a finite interval, Russ. Math. Surv. 35 (1980), 81-135. 